\title{
Two forms of spiral-wave reentry in an ionic model of ischemic ventricular myocardium
}

\author{
Aoxiang Xu and Michael R. Guevara \\ Department of Physiology and Centre for Nonlinear Dynamics in Physiology and Medicine, \\ McGill University, 3655 Drummond Street, Montréal, Québec, H3G 1 Y6 Canada
}

(Received 28 July 1997; accepted for publication 25 November 1997)

\begin{abstract}
It is well known that there is considerable spatial inhomogeneity in the electrical properties of heart muscle, and that the many interventions that increase this initial degree of inhomogeneity all make it easier to induce certain cardiac arrhythmias. We consider here the specific example of myocardial ischemia, which greatly increases the electrical heterogeneity of ventricular tissue, and often triggers life-threatening cardiac arrhythmias such as ventricular tachycardia and ventricular fibrillation. There is growing evidence that spiral-wave activity underlies these reentrant arrhythmias. We thus investigate whether spiral waves might be induced in a realistic model of inhomogeneous ventricular myocardium. We first modify the Luo and Rudy [Circ. Res. 68, 1501-1526 (1991)] ionic model of cardiac ventricular muscle so as to obtain maintained spiral-wave activity in a two-dimensional homogeneous sheet of ventricular muscle. Regional ischemia is simulated by raising the external potassium concentration $\left(\left[\mathrm{K}^{+}\right]_{o}\right)$ from its nominal value of $5.4 \mathrm{mM}$ in a subsection of the sheet, thus creating a localized inhomogeneity. Spiral-wave activity is induced using a pacing protocol in which the pacing frequency is gradually increased. When $\left[\mathrm{K}^{+}\right]_{o}$ is sufficiently high in the abnormal area (e.g., $20 \mathrm{mM}$ ), there is complete block of propagation of the action potential into that area, resulting in a free end or wave break as the activation wave front encounters the abnormal area. As pacing continues, the free end of the activation wave front traveling in the normal area increasingly separates or detaches from the border between normal and abnormal tissue, eventually resulting in the formation of a maintained spiral wave, whose core lies entirely within an area of normal tissue lying outside of the abnormal area ("type I" spiral wave). At lower $\left[\mathrm{K}^{+}\right]_{o}$ (e.g., $10.5 \mathrm{mM}$ ) in the abnormal area, there is no longer complete block of propagation into the abnormal area; instead, there is partial entrance block into the abnormal area, as well as exit block out of that area. In this case, a different kind of spiral wave (transient "type II" spiral wave) can be evoked, whose induction involves retrograde propagation of the action potential through the abnormal area. The number of turns made by the type II spiral wave depends on several factors, including the level of $\left[\mathrm{K}^{+}\right]_{o}$ within the abnormal area and its physical size. If the pacing protocol is changed by adding two additional stimuli, a type I spiral wave is instead produced at $\left[\mathrm{K}^{+}\right]_{o}=10.5 \mathrm{mM}$. When pacing is continued beyond this point, apparently aperiodic multiple spiral-wave activity is seen during pacing. We discuss the relevance of our results for arrythmogenesis in both the ischemic and nonischemic heart. (C) 1998 American Institute of Physics. [S1054-1500(98)02501-4]
\end{abstract}

Cardiovascular disease is the leading cause of death in the industrialized world. In many individuals, death is caused by a transient disturbance in the normal rhythm of the heartbeat ("cardiac arrhythmia"). The tragic fact in many of these cases is that the heart muscle is not yet irreversibly damaged when the arrhythmia starts upprompt treatment to abolish the arrhythmia soon enough can lead to a successful outcome. The two most dangerous arrhythmias encountered are ventricular tachycardia, when the ventricles of the heart contract too rapidly, and ventricular fibrillation, when the individual ventricular cells contract in an asynchronous fashion. Both of these arrhythmias are almost always due to waves of electrical activity circulating in the ventricular muscle ("reentry"). It has been suggested recently that spiral waves ("rotors," "reverberators," "vortices"), a form of selfsustained reentrant activity that has been described in many excitable media, might be responsible for these arrhythmias. However, experimental and modeling work on spiral waves in cardiac muscle has, so far, largely dealt with homogeneous systems. Since inhomogeneities are known to play a role in the induction of reentrant rhythms in normal hearts as well as in diseased hearts, we investigate a model of an inhomogeneous sheet of ventricular muscle. We find that two different forms of spiral-wave activity can be induced. In both cases, the presence of the inhomogeneity is critical in initiating the spiral-wave activity. However, in only one of the two cases is the presence of the inhomogeneity crucial in maintaining the spiral-wave activity. 


\section{INTRODUCTION}

Cardiac arrhythmias such as ventricular tachycardia and ventricular fibrillation can have very serious consequences for an individual. These malignant arrhythmias are almost always due to self-perpetuating circulating wave fronts of electrical activity within the ventricular muscle. ${ }^{1-4}$ The classic "head-eat-tail" circus movement type of reentry ${ }^{5-8}$ has historically been assumed to be the mechanism underlying such tachyarrhythmias. In a circus movement, the electrical activity basically travels around a one-dimensional ringshaped structure, with the circumference of the ring being sufficiently long so that when the wave front encounters the starting point once again, that area will have recovered to an extent that allows the wave front to successfully traverse that area once again (i.e., 'reenter'). In recent years, it has been hypothesized that a different form of reentry-spiral waves (also called rotors, vortices, autowaves, reverberators)—may instead be the form of reentrant activity responsible for generating these arrhythmias. ${ }^{9-15}$ For example, monomorphic ventricular tachycardia might be produced by a spiral wave with a stationary core, ${ }^{9,10,16}$ and polymorphic ventricular tachycardia by a drifting spiral wave. ${ }^{17-19}$ Ventricular fibrillation might be associated with a single drifting spiral ${ }^{20,21}$ or multiple coexisting spiral waves. ${ }^{22-24}$ There is also now some experimental evidence for the existence of spiral waves in cardiac tissue. ${ }^{18,20,25-31}$ While there have been many studies showing that spiral-wave activity is generic in simplified models of excitable media (see references in Ref. 32), there have been fewer descriptions of spiral-wave activity in twodimensional sheets of ventricular muscle modeled by realistic ionic models..$^{17,19,30,33-42}$ In addition, all of this modeling has been for homogeneous sheets of muscle: i.e., the cells making up the tissue are assumed to be identical and to be connected to one another in a uniform way. However, it has been known for many years that normal ventricular myocardium is quite inhomogeneous, with large-scale gradients in electrical properties from the apex to the base of the ventricles $^{43,44}$ and from endocardium to epicardium, ${ }^{43,45}$ as well as more localized, smaller-scale inhomogeneities. ${ }^{46}$ There is, in addition, a systematic rotation of the fiber direction as the myocardium is traversed from epicardium to endocardium. ${ }^{41}$ Many of the interventions that make it easier to induce ventricular arrhythmias also increase the degree of inhomogeneity present-e.g., stimulation of the sympathetic nervous system, ${ }^{46,47}$ hypothermia, ${ }^{46,48}$ premature stimulation, ${ }^{46}$ fast pacing, ${ }^{44}$ and various drugs. ${ }^{46}$ In fact, it is widely believed that there is a causal relationship between this increased inhomogeneity ("increased dispersion of refractoriness") and the increased incidence of arrhythmias. In some of these examples, the inhomogeneity is local, in the sense that it is the dispersion in the electrophysiological properties of cells within a relatively small neighborhood around a given point that is increased, and comparable increases are seen at other points in the ventricle.

Myocardial ischemia occurs when the blood supply to the heart muscle is not sufficient to meet its metabolic demands. Should ischemia be pronounced enough and last for a sufficiently long time, heart muscle will die ("myocardial infarction"). The most common cause of myocardial infarction is the occlusion of a stenosed coronary artery by a clot. Ventricular arrhythmias frequently occur during the acute stage of myocardial infarction. There is overwhelming experimental evidence that these arrhythmias are due to altered electrophysiological properties caused by hypoxia, acidosis, increased extracellular potassium concentration ("hyperkalemia"), etc. (see Ref. 49 for a review). These include depolarization of the resting membrane potential, fall in overshoot potential and amplitude, shortening of the action potential duration, slowing of the upstroke velocity and conduction velocity, and the appearance of post-repolarization refractoriness. Thus the inhomogeneity, which in this case is localized to a very specific area of the ventricle, once again plays a leading role in arrhythmogenesis. We therefore decided to investigate the effect of introducing a localized inhomogeneity representing the ischemic area into an otherwise homogeneous sheet of ventricular myocardium. The extracellular potassium concentration was raised within that area to create the inhomogeneity.

\section{METHODS}

\section{Choice of ionic model}

A fundamental decision to be taken upon embarking on modeling of reentry is the choice of ionic model, since there are now many ionic models of ventricular muscle available. ${ }^{50-62}$ The first seven of these papers describe "firstgeneration" models, all of which are modifications of the original Beeler-Reuter (BR) model. ${ }^{50}$ They are much simpler that the latter six "second-generation" models. The major difference is that the second-generation models have concentrations of several of the ionic species that vary in time (rather than stay fixed), internal $\mathrm{Ca}^{++}$dynamics (involving $\mathrm{Ca}^{++}$uptake by and release from sarcoplasmic reticulum), and $\mathrm{Na}^{+}-\mathrm{K}^{+}$pump and $\mathrm{Na}^{+}-\mathrm{Ca}^{++}$exchange currents. We decided against using a second-generation model for two main reasons. First, there are problems of flux balance with the second-generation class of models that can lead to slow drifts in the concentrations of ions. ${ }^{63}$ For example, prolonged periodic stimulation at a BCL of $1000 \mathrm{~ms}$ of the secondgeneration atrial model ${ }^{64}$ leads to a continuing depletion of internal $\left[\mathrm{K}^{+}\right]$in time that shows no sign of asymptoting after $1000 \mathrm{~s}$ of simulation (Xu and Guevara, unpublished). While there have been recently a few papers published showing reentrant activity in second-generation models, ${ }^{37,39,40,65,66}$ we have chosen not to work with models that show such drifts at the present time, because of the risk that these slow drifts might produce artefactual results (e.g., slow changes in refractory period or in conduction velocity leading to wave front separation) in long-lasting simulations, such as our own, in which many beats are involved. Second, since the investigation of reentry in ionic models is relatively new, it is perhaps prudent to first investigate simpler models, where it will be easier to gain insight into the fundamental ionic mechanisms involved.

The question now arises of which of the first-generation models to use. The original "first-generation" ventricular 
model, the BR model, ${ }^{50}$ which has been used in several papers modeling spiral waves ${ }^{19,33,36,38}$ has an upstroke velocity of the action potential of $\sim 100 \mathrm{~V} \mathrm{~s}^{-1} .50,67$ Because of the inhomogeneity of the heart, the experimentally reported in situ values span a wide range. In one report on guinea-pig ventricle, the average value in epicardial muscle was $110 \mathrm{~V} \mathrm{~s}^{-1}$, while the average value in endocardial muscle was $248 \mathrm{~V} \mathrm{~s}^{-1} .^{43} \mathrm{~A}$ value as high as $\sim 400 \mathrm{~V} \mathrm{~s}^{-1}$ has been estimated using an optical fluorescence method. ${ }^{68}$ The BR value is thus at the lower end of the experimentally reported range. The Luo and Rudy (LR) model ${ }^{55}$ has a maximum upstroke velocity of $\sim 300 \mathrm{~V} \mathrm{~s}^{-1}$, which is toward the upper end of the experimentally reported range. The LR model is among the more recent of the first-generation models, and has thus far been used in about a score of modeling studies. ${ }^{29,69,70}$ In addition, it allows for alteration of the external potassium concentration $\left(\left[\mathrm{K}^{+}\right]_{o}\right)$, which is the parameter we change to simulate the effect of ischemia. We therefore chose the LR model as our basic model in these, our first, simulations of reentry in an ionic model of ischemic muscle. To obtain persistent spiral-wave activity, we modify the LR equations by changing three parameters away from their standard values (see the Results section). Unless stated otherwise, we use the nominal value $\left[\mathrm{K}^{+}\right]_{o}=5.4 \mathrm{mM}$ used in Luo and Rudy $(1991)^{55}$ that is close to the physiological value.

While, as noted above, there have been many studies published using the LR model, there are inconsistencies between the results presented in Luo and Rudy $(1991)^{55}$ and the equations given in Table I of that paper. Using the equations in the table, one does not obtain the results shown in Fig. 2 of the paper, showing that there is a discrepancy with respect to the inwardly rectifying potassium current $\left(I_{\mathrm{K} 1}\right)$. Since initial conditions were not given, it is not possible to verify the results shown in the other figures of that paper (with the exception of Fig. 1, which agrees with the results we obtain using the equations in Table 1). In our work, we have used the equations appearing in Table 1 of Luo and Rudy (1991). ${ }^{55}$

\section{Numerical methods}

We simulate a two-dimensional sheet of isotropic ventricular muscle using the cable equations

$$
\left(\frac{\partial^{2} V}{\partial x^{2}}+\frac{\partial^{2} V}{\partial y^{2}}\right)=\rho S_{v}\left(C_{m} \frac{\partial V}{\partial t}+I_{\text {ion }}\right),
$$

where $V$ is the transmembrane potential $(\mathrm{mV}), I_{\text {ion }}$ is the total ionic current $\left(\mu \mathrm{A} \mathrm{cm}^{-2}\right), t$ is time (ms), $x$ and $y$ are spatial coordinates in the sheet $(\mathrm{cm}), C_{m}$ is the specific membrane capacitance $\left(1 \mu \mathrm{F} \mathrm{cm}^{-2}\right), \rho$ is the bulk cytoplasmic resistivity $(0.2 \mathrm{k} \Omega \mathrm{cm}), \quad S_{v}$ is the surface-to-volume ratio $\left(5000 \mathrm{~cm}^{-1}\right)$. An explicit Euler scheme is used for the numerical integration of Eq. (1),

$$
\begin{aligned}
& \frac{V_{i-1, j}(t)-2 V_{i, j}(t)+V_{i+1, j}(t)}{\Delta x^{2}} \\
& +\frac{V_{i, j-1}(t)-2 V_{i, j}(t)+V_{i, j+1}(t)}{\Delta y^{2}} \\
& =\rho S_{v} C_{m} \frac{V_{i, j}(t+\Delta t)-V_{i, j}(t)}{\Delta t}+\rho S_{v} I_{\text {ion }}(t),
\end{aligned}
$$

where $V_{i j}$ is the voltage of the element located at node $(i, j)$, $\Delta x$ and $\Delta y$ are the spatial step sizes, and $\Delta t$ is the temporal step size. In iterating from time $t$ to time $(t+\Delta t), I_{\text {ion }}$ in Eq. (2) is calculated from $V(t)$ and the activation and inactivation variables $\xi_{i}$ evaluated at time $t+\Delta t$,

$$
\xi_{i}(t+\Delta t)=\xi_{i}(\infty)-\left[\xi_{i}(\infty)-\xi_{i}(t)\right] e^{-\Delta t / \tau_{i}},
$$

where $\xi_{i}(\infty)$ is the steady-state or asymptotic value of $\xi_{i}$ and $\tau_{i}$ is the time constant of $\xi_{i}$, both of which are functions of $V$. Both $\xi_{i}(\infty)$ and $\tau_{i}$ are stored in a look-up table using increments in $V$ of $0.2 \mathrm{mV}$, and linear interpolation is used to obtain the values on the right-hand side of Eq. (3). A simple forward Euler scheme is used to evaluate the internal calcium concentration $\left(\left[\mathrm{Ca}^{++}\right]_{i}\right)$,

$$
\left[\mathrm{Ca}^{++}\right]_{i}(t+\Delta t)=\left[\mathrm{Ca}^{++}\right]_{i}(t)+\frac{d\left[\mathrm{Ca}^{++}\right]_{i}(t)}{d t} \Delta t .
$$

We use a $100 \times 100$ grid, with $\Delta x=\Delta y=0.025$ $\mathrm{cm} .{ }^{29,33,36,38}$ The sheet size is thus $2.5 \mathrm{~cm} \times 2.5 \mathrm{~cm}$. The space constant $(\lambda)$ here at $\left[\mathrm{K}^{+}\right]_{o}=5.4 \mathrm{mM}$ is $0.6 \mathrm{~mm}$, calculated from the formula for a one-dimensional cable $[\lambda$ $=\left(R_{m} / \rho S_{v}\right)^{1 / 2}$, where $R_{m}$ is the membrane resistance $(3.55$ $\left.\left.\mathrm{k} \Omega \mathrm{cm}^{2}\right)\right]$. We use an integration step size $\Delta t$ of 0.025 ms. ${ }^{33,38}$ With these parameters, the linear stability criterion for the explicit Euler scheme, which is $(\Delta x)^{2} / \Delta t>4 D$, where $D=\left(\rho S_{v} C\right)^{-1}=10^{-3} \mathrm{~cm}^{2} \mathrm{~ms}^{-1}$ is the diffusion coefficient, is satisfied. ${ }^{71}$ Using our integration scheme, the maximum upstroke velocity is $300 \mathrm{~V} \mathrm{~s}^{-1}$ for a propagated planar wave front in an infinitely rested sheet. Neumann boundary conditions (i.e., zero-current flux across edge of sheet) are used. Stimulus pulses are $1 \mathrm{~ms}$ in duration, $300 \mu \mathrm{A} \mathrm{cm}^{-2}$ in amplitude $(\sim$ twice the diastolic threshold), and applied to the leftmost column of the sheet, unless otherwise stated.

Numerical integration of Eq. (1) using Eqs. (2) -(4) was carried out on an SGI Indigo-2 workstation using programs written in C (16 significant decimal places). Data was stored at $5 \mathrm{~ms}$ intervals in a disk file, unless indicated otherwise. Black-and-white or colour voltage maps (GIF or PPM format) were constructed from this data and downloaded to a Pentium-based PC. The figures shown below were obtained by printing out the black-and-white GIF files on a laser printer (1200 dpi). The PPM files were made into MPEG-format movies using the program CMPEG (version 1.0). The original color movies can be obtained from http:// www.physio.mcgill.ca/guevaralab or http://www.aip.org/ epaps/epaps.html. ${ }^{145}$ 

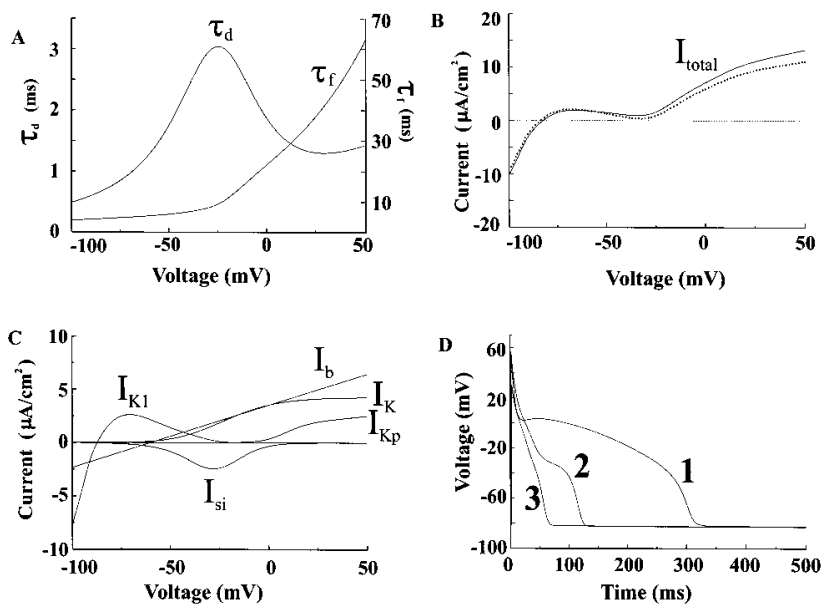

FIG. 1. (a) Time constants for activation $\left(\tau_{d}\right)$ and inactivation $\left(\tau_{f}\right)$ of the slow inward current $\left(I_{\mathrm{si}}\right)$ in the modified LR model. (b) Steady-state current-voltage (IV) relationship of total current in original (broken curve) and modified (solid curve) LR models. (c) Steady-state IV relationships of individual ionic currents in modified LR model. (d) Space-clamped action potentials in the original LR model (trace 1) and in our modified LR model (trace 2) from infinite-rest initial conditions. Trace 3 shows action potential in the modified model in steady state at $\mathrm{BCL}=75 \mathrm{~ms}$ ( $41 \mathrm{st}$ action potential shown after the start of the simulation from infinite-rest conditions). Pulse amplitude $=50 \mu \mathrm{A} \mathrm{cm}^{-2}$, pulse duration $=1 \mathrm{~ms}$.

\section{RESULTS}

\section{Modification of LR model}

Reentrant motion consistent with spiral-wave reentry has been described in the intact ventricle ${ }^{18,20,26}$ as well as in thin slices of ventricular muscle a few $\mathrm{cm}^{2}$ in area. ${ }^{27,28,30,72}$ As with many other simulations using unmodified ventricular models, ${ }^{19,33,35,36,38}$ we have not been able to obtain maintained spiral waves in our simulations with a $2.5 \times 2.5 \mathrm{~cm}$ sheet using the standard LR model. This might be due to the wavelength (the product of conduction velocity and refractory period) being too large (perhaps with respect to the sheet size), as discussed later. Therefore, we have found it necessary to modify the LR model in order to obtain persistent spiral-wave activity.

Perhaps the major deficiency of the LR model, which is a modification of the BR model, is in its description of the slow inward calcium current $I_{\mathrm{si}}$, which was carried over unchanged from the BR model. It has been known for a long time that the time constants of activation $\left(\tau_{d}\right)$ and inactivation $\left(\tau_{f}\right)$ are unrealistically long in the BR model, being much larger in the model than in the experiment. ${ }^{73,74} \mathrm{We}$ thus divide both of these time constants by a factor of 12 in order to bring them closer to the experimentally described values. This strategy has been previously used to obtain persistent spiral-wave activity in models of the BR type. ${ }^{33,35,38,42}$ The modified $\tau_{d}$ and $\tau_{f}$ curves [Fig. 1(a)] are then more consistent with the experimental data (experiment: $\tau_{d}$ $=2-5 \mathrm{~ms}, \tau_{f}=10-50 \mathrm{~ms}$ (Refs. 74 and 75); model: $\tau_{d}$ $=2.7 \mathrm{~ms}$ at $-35 \mathrm{mV}$ and $1.5 \mathrm{~ms}$ at $10 \mathrm{mV}, \tau_{f}=11.4 \mathrm{~ms}$ at $-20 \mathrm{mV}$ and $28.3 \mathrm{~ms}$ at $10 \mathrm{mV}$ ). However, with this modification, the voltage then "hangs up" in the plateau range of potentials, so that the action potential duration (APD) remains essentially unchanged. To reduce the APD so as to allow sustained spiral-wave activity, we are forced to made a second modification, which is to multiply the background current $\left(I_{b}\right)$ by a factor of 1.5 , resulting in more repolarizing current in the plateau range of potentials [Figs. 1(b) and 1 (c)]. Further justification for modifying $I_{b}$, in particular, at this stage can be found in the Discussion below. The steadystate current voltage (IV) relationship in the modified model [the solid curve in Fig. 1(b)] is then not too different from that of the unmodified model (broken curve), resembling that typical of ventricular muscle. ${ }^{76}$ Figure $1(\mathrm{~d})$ shows that the space-clamped action potential from infinite-rest initial conditions is markedly reduced in duration in our modified LR model (trace 2) with respect to the original LR model (trace $1)$.

\section{Restitution, dispersion, and wavelength in homogeneous sheet}

For any sort of sustained reentrant motion (e.g., circus movement, spiral wave) to exist, it is clear that an activated area must have time to recover ("come out of refractoriness") before it is invaded once again., ${ }^{5,7}$ In a classic "head-eat-tail" circus-movement circuit, ${ }^{7,8,77,78}$ the minimal pathlength that will theoretically allow this to occur is thus given by the product of the conduction velocity and the minimum time possible between two successive activations, which is termed the refractory period. This minimal pathlength is called the wavelength $(\lambda)$. The two most important parameters controlling reentry are thus the conduction velocity and the refractory period. [Note that wavelength as defined here is the wavelength of a planar wave, and not the wavelength of a spiral wave ("spiral wavelength").]

It is clear from prior modeling work in simplified excitable media that the circus-movement and spiral-wave forms of reentry are related. ${ }^{79}$ It has been found that the wavelength measured during fast pacing is comparable to the size of the reentrant circuit in "leading-circle", reentry, ${ }^{78}$ which was initially described in atrial muscle ${ }^{77}$ and subsequently identified as spiral wave in origin. ${ }^{14,15,66}$ Both the circus-movement ${ }^{80}$ and spiral-wave (e.g., Ref. 27) forms of reentry can produce tachycardia. It is well known in ventricular muscle that decreasing the interval between stimuli (the basic cycle length or BCL) usually results in a fall in the APD and the refractory period [compare trace 3 $(\mathrm{BCL}=75 \mathrm{~ms})$ with trace $2(\mathrm{BCL}=\infty)$ in Fig. $1(\mathrm{~d})]$, as well as in the conduction velocity. ${ }^{80,81}$ Both of these changes contribute to a fall in wavelength. Since we shall be producing spiral waves by systematically decreasing the BCL, we therefore made a systematic survey of how APD, conduction velocity, and wavelength are influenced by $\mathrm{BCL}$ in our modified LR model.

A homogeneous sheet was first paced at $\mathrm{BCL}=1000 \mathrm{~ms}$. The APD and conduction velocity were measured in the center of the sheet. The wavelength was then calculated, replacing the refractory period with APD, since the two are very close. The BCL was then systematically lowered in a stepwise fashion, until propagation failed at $\mathrm{BCL}=69 \mathrm{~ms}$. Figure 2(a) shows the APD restitution curve, Fig. 2(b) shows the velocity dispersion curve, and Fig. 2(c) shows the wavelength curve. At the shorter BCLs, the curves in Fig. 2 are all 


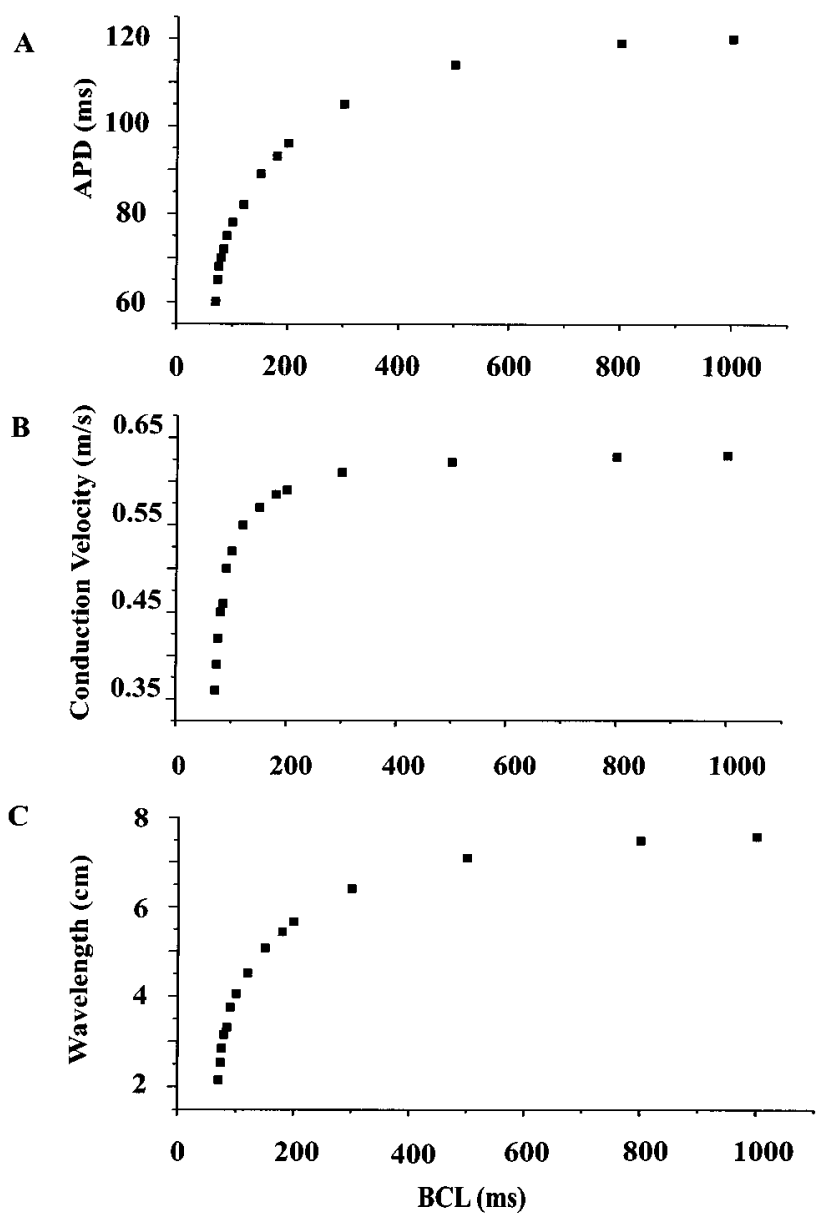

FIG. 2. (a) Action-potential duration (APD) restitution curve. APD defined as the time from $-20 \mathrm{mV}$ on the upstroke of an action potential to $-80 \mathrm{mV}$ on the repolarizing limb of action potential. (b) Velocity dispersion curve. Conduction velocity calculated from the time between the onset of a stimulus pulse and crossing of $-20 \mathrm{mV}$ in the element in the center of the sheet. (c) Wavelength curve. Computed as the product of APD and velocity. Initial conditions obtained by setting variables at each element of the sheet to steady-state values appropriate to a space-clamped cell at $t=-1000 \mathrm{~ms}$, and then leaving the sheet to asymptotically approach the steady state over the next $1000 \mathrm{~ms}$. Pacing protocol: $\mathrm{BCL}=1000 \mathrm{~ms}$ (three cycles delivered), 800-300 s (two cycles at each BCL), 200-70 (five cycles at each BCL). Stimuli [current pulses of duration $1 \mathrm{~ms}$ and amplitude $300 \mu \mathrm{A} \mathrm{cm}^{-2}$ ( $\sim$ twice the diastolic threshold amplitude)] applied to the column of elements at the left edge of the sheet. Data points in this simulation are stored every $1.0 \mathrm{~ms}$.

very close to the curves found in guinea-pig ventricular epicardium at an external calcium concentration $\left(\left[\mathrm{Ca}^{++}\right]_{0}\right)$ of $1.25 \mathrm{mM}^{80}$

\section{Spiral wave in a homogeneous sheet of muscle}

Our modifications to the LR model result in a reduction of APD and wavelength, thus permitting persistent spiralwave activity in our $2.5 \mathrm{~cm} \times 2.5 \mathrm{~cm}$ sheet. Figures $3(\mathrm{a})-3(\mathrm{~h})$ show a spiral wave induced by a modified crossed-gradient protocol, ${ }^{15-17}$ in which the left edge of the sheet is stimulated at $t=0 \mathrm{~ms}$ with the first stimulus $\left(\mathrm{S}_{1}\right)$, and the entire upperleft quadrant ${ }^{35}$ is stimulated at $t=180 \mathrm{~ms}$ with a second stimulus $\left(\mathrm{S}_{2}\right)$. The period of rotation of the spiral wave is $\sim 75 \mathrm{~ms}$, with the action potential sufficiently away from the core (the central area not activated on a given turn of the spiral wave) resembling that shown in Fig. 1(d) (trace 3).

While the spiral wave persisted to the end of the computation $(t=5000 \mathrm{~ms})$, there was meander in the position of its tip. The position of the tip was estimated by eye, and Figs. 3(i) -3(1) show the trajectory of the tip for several successive turns of the spiral wave midway through the computation. With the exception of a brief transient probably due to initial conditions, the tip stayed in the same general area visited in Fig. 3 for the $\sim 70$ turns that the spiral wave made during the $5000 \mathrm{~ms}$ simulation.

\section{Type I spiral wave in an inhomogeneous sheet of muscle}

As mentioned in the Introduction, ischemia results in depolarization of the resting membrane potential, fall in overshoot potential and amplitude, shortening of the APD, slowing of the upstroke velocity and conduction velocity, and the appearance of post-repolarization refractoriness. Perhaps the single most important factor in producing these alterations in the action potential is the ischemia-induced elevation of $\left[\mathrm{K}^{+}\right]_{o}{ }^{82-85}$ We therefore model the effect of regional ischemia by simply raising $\left[\mathrm{K}^{+}\right]_{o}$ in a delimited subsection of an otherwise homogeneous sheet.

Figure 4 shows the voltage in a resting inhomogeneous sheet incorporating a $0.75 \mathrm{~cm} \times 0.75 \mathrm{~cm}$ ischemic area. This simulated ischemic area is visible at the bottom of the sheet, and is lighter in color than the normal area because the resting potential is depolarized to about $-50 \mathrm{mV}$ as a consequence of $\left[\mathrm{K}^{+}\right]_{o}$ being set equal to $20 \mathrm{mM}$ in that area. In contrast, the resting potential in the normal area $\left(\left[\mathrm{K}^{+}\right]_{o}=5.4 \mathrm{mM}\right)$ is $-87 \mathrm{mV}$. We chose $20 \mathrm{mM}$ since this is toward the upper limit of the range measured in the ischemic area during the acute phase of experimental myocardial ischemia. ${ }^{82}$ Due to the existence of electrotonic interactions, we obtained approximate initial conditions by the following procedure: at $t=-1000 \mathrm{~ms}$, variables for each element in the normal (resp., ischemic) area were set equal to the steadystate space-clamped values appropriate to $\left[\mathrm{K}^{+}\right]_{o}=5.4 \mathrm{mM}$ (resp., $20 \mathrm{mM}$ ). The simulation of the sheet was then run for $1000 \mathrm{~ms}$, to allow electrotonic interactions to occur. This produces a spatially extended border zone centered on the demarcation line between normal and ischemic tissue (Fig. 4 shows the situation at $t=0 \mathrm{~ms}$ ). Within this border zone (effectively a few space constants wide), there is a spatial gradient in the voltage and other variables. The gradient in voltage is manifested in the fuzziness of the borders of the ischemic area in Fig. 4. The values of the variables describing the state of each element in the sheet at $t=0 \mathrm{~ms}$ were then taken as initial conditions for the simulations we shall now present.

It has been known for a long time that electrical stimulation can be used to provoke tachyarrhythmias such as tachycardia and fibrillation in both healthy and ischemic hearts. Indeed, there are several protocols by which this is routinely accomplished in patients undergoing electrophysiological investigation of their arrhythmias: e.g., a single extrastimulus $\left(S_{1}-S_{2}\right.$ protocol, such as that used in Fig. 3), multiple extrastimuli (e.g., $S_{1}-S_{2}-S_{3}$ protocol), ramp pacing 

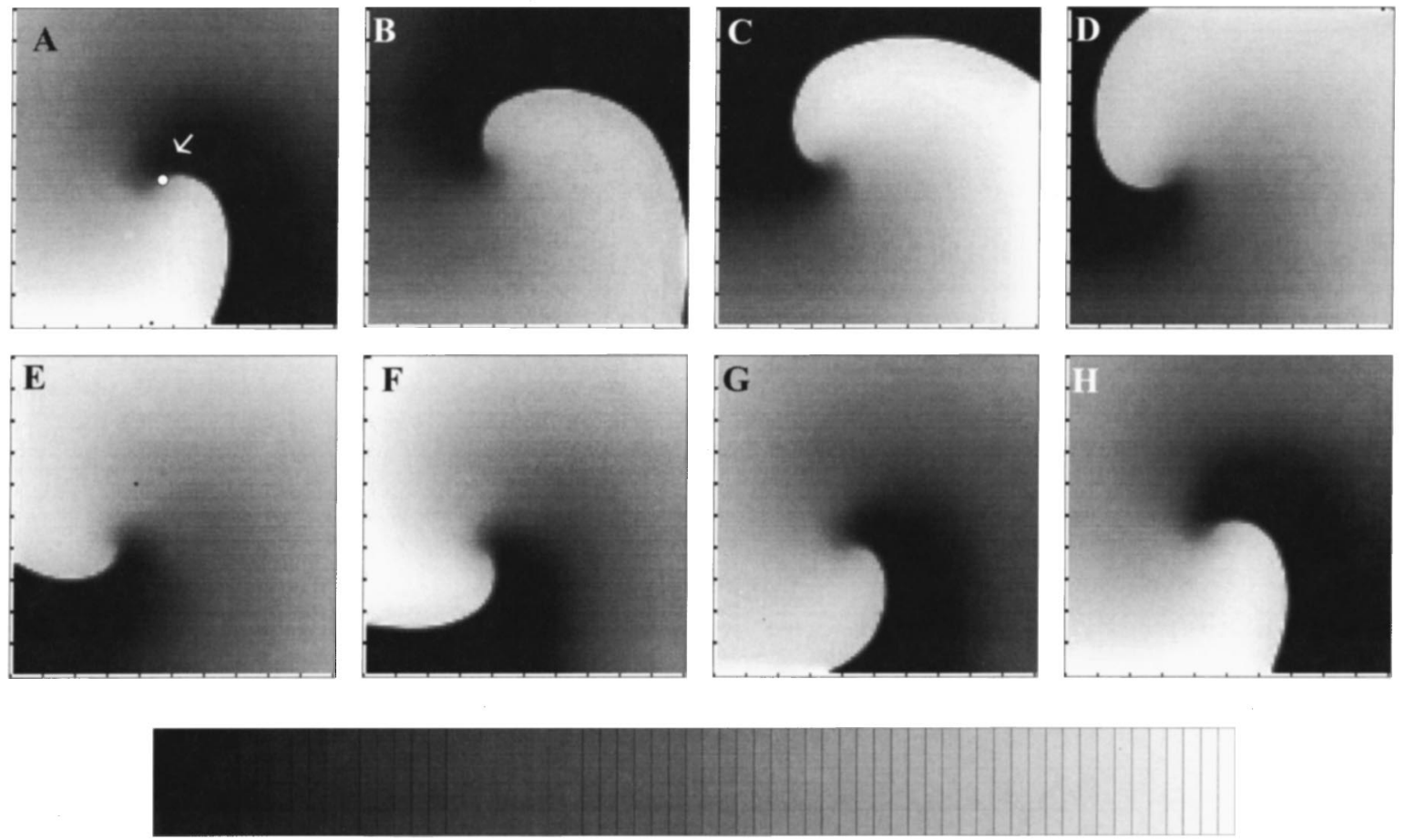

\section{$-90 \mathrm{mV}$}
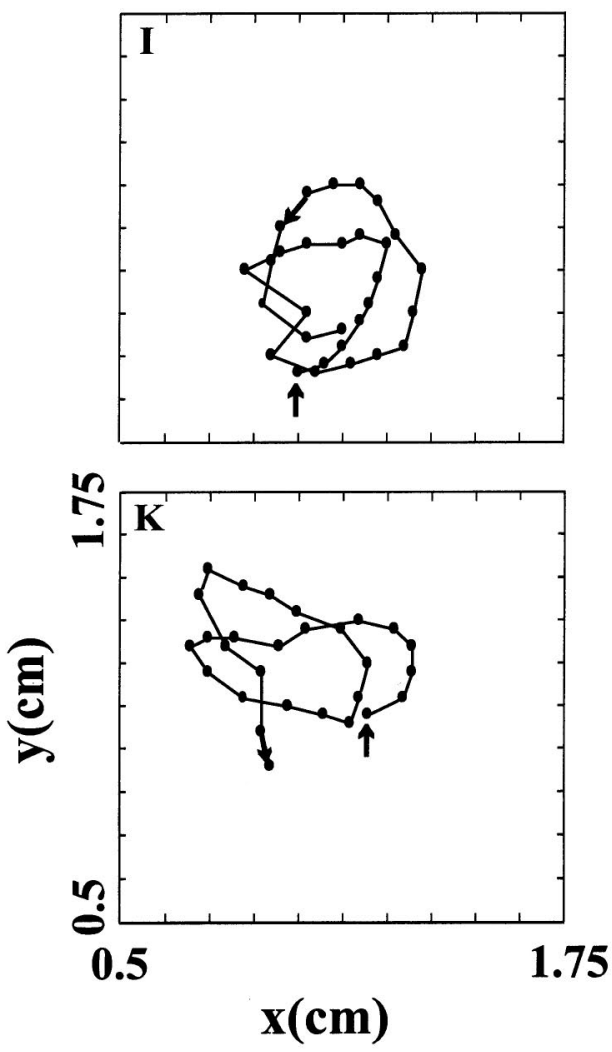

$+20 \mathrm{mV}$
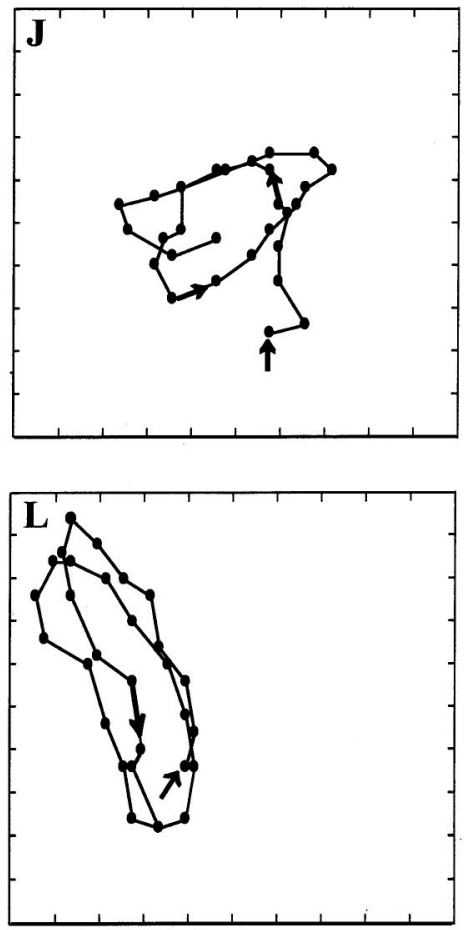

FIG. 3. (a)-(h) One rotation of a spiral wave in a homogeneous sheet described by modified LR model. Time after the start of the simulation: (a) 910 ms, (b) $930 \mathrm{~ms}$, (c) $940 \mathrm{~ms}$, (d) $950 \mathrm{~ms}$, (e) $960 \mathrm{~ms}$, (f) $970 \mathrm{~ms}$, (g) $980 \mathrm{~ms}$, and (h) $990 \mathrm{~ms}$. The spiral wave is induced by a two-pulse ( $\left.\mathrm{S}_{1}-\mathrm{S}_{2}\right) \mathrm{crossed}-\mathrm{gradient}$ protocol, with the first stimulus $\left(\mathrm{S}_{1}\right)$ delivered to the leftmost column of elements at $t=0 \mathrm{~ms}$, and a second stimulus $\left(\mathrm{S}_{2}\right)$ delivered to all elements in the upper-left quadrant of the sheet at $t=180 \mathrm{~ms}$. Stimuli are current pulses of duration $1 \mathrm{~ms}$ and amplitude $300 \mu \mathrm{A} \mathrm{cm}{ }^{-2}(\sim$ twice the diastolic threshold amplitude). The same initial conditions are used as in Fig. 2. The gray scale at the bottom gives transmembrane potential. (i)-(1): Tip trajectory (estimated by hand-and-eye) plotted every $5 \mathrm{~ms}$ (filled circles). First point plotted is for $t=2000 \mathrm{~ms}$ in (i). The white circle indicated by the arrow in panel (a) above indicates estimate of the tip-position. Arrows in each panel indicate starting point and direction of trajectory [data is continuous from (i) through (1)]. Note that only a part of the entire sheet is shown. 


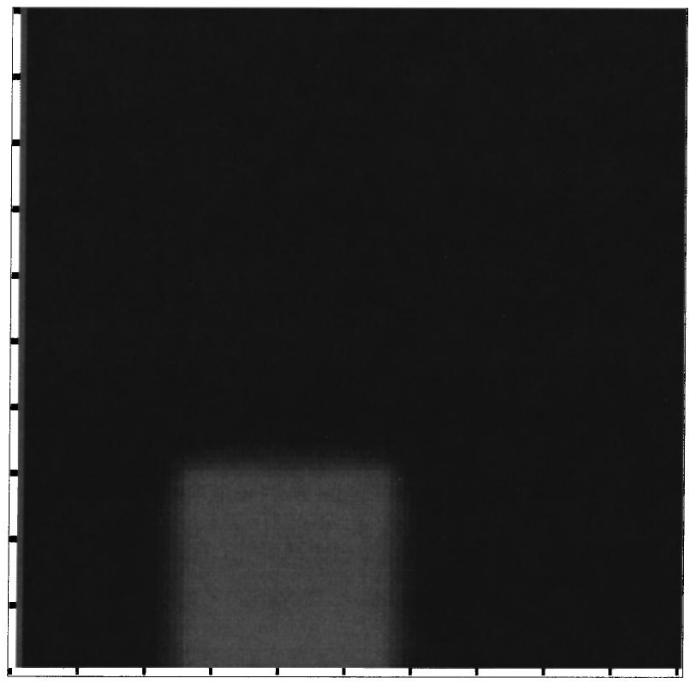

FIG. 4. Resting potential in an inhomogeneous sheet containing simulated ischemic area with $\left[\mathrm{K}^{+}\right]_{o}=20 \mathrm{mM}$ (the lighter area at the bottom of the sheet). In the rest of the sheet ("normal area"), $\left[\mathrm{K}^{+}\right]_{o}=5.4 \mathrm{mM}$. Initial conditions obtained as described in the text.

(gradually decreasing BCL), decremental pacing (a stepwise decrease in BCL), and burst pacing (a few stimuli at a very short BCL). Here we use decremental pacing, which will result in a progressive reduction in the wavelength as BCL is decreased [Fig. 2(c)], and so promote the induction of reentrant motions.

The resting inhomogeneous sheet of Fig. 4 is periodically paced with a train of current pulses applied to the lefthand row of elements in the sheet. The BCL is held constant for 1 to 20 pacing intervals, and then suddenly stepped to a shorter interval. Figures $5(\mathrm{a})-5(\mathrm{~d})$ show the propagation of the action potential elicited by the first stimulus. The $\left[\mathrm{K}^{+}\right]_{o}$ is sufficiently high $(20 \mathrm{mM})$ so that there is block of propagation of the action potential into the ischemic area [Fig. 5(a)]. The activation wave front thus breaks as it encounters the ischemic area, developing a "free end" or "broken end" that attaches or anchors ${ }^{86}$ to the border between normal and ischemic tissue [Figs. 5(a) and 5(b)]. This free end, which somewhat resembles a spiral tip (arrow in Fig. 5(b)], curves around the inexcitable obstacle [Fig. 5(b)] and propagates out of the bottom of the sheet. Repolarization starts at the left-hand border of the ischemic area [Fig. 5(c)] and the repolarization wave front then spreads out in a more-or-less radial direction from the ischemic area [Fig. 5(d)].

The BCL was initially $180 \mathrm{~ms}$, and was reduced stepwise to $150,120,100,90,80,74$, and finally $71 \mathrm{~ms}$. Figures $5(\mathrm{e})-5(\mathrm{~h})$ show the propagation of the activation wave front of the third action potential at $\mathrm{BCL}=71 \mathrm{~ms}$. The conduction velocity is slowed with respect to that of the first action potential [Figs. 5(a)-5(d)] because of the presence now of a rather short excitable gap between this action potential and the immediately preceding one [Figs. $5(\mathrm{e})-5(\mathrm{~g})]$. As with the first action potential [Figs. 5(a) and 5(b)], the most curved part of the wave front-again resembling a spiral tip-firmly anchors within the border zone between the normal and ischemic areas as the action potential propagates along the top
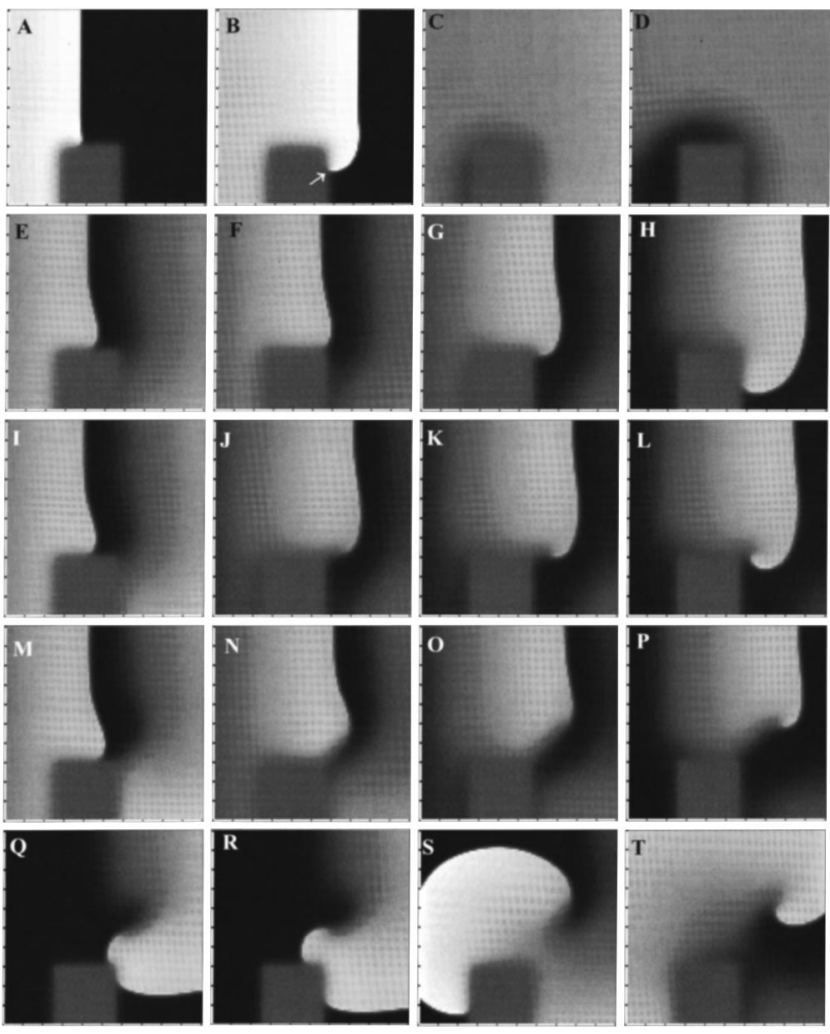

FIG. 5. Type I spiral wave in an inhomogeneous sheet $\left(\left[\mathrm{K}^{+}\right]_{o}=20 \mathrm{mM}\right.$ in the ischemic area). Spiral wave (type I) produced by the decremental pacing protocol. See the text for a further description. Time after the start of the simulation: (a) $15 \mathrm{~ms}$, (b) $30 \mathrm{~ms}$, (c) $95 \mathrm{~ms}$, (d) $120 \mathrm{~ms}$, (e) $2765 \mathrm{~ms}$, (f) $2775 \mathrm{~ms}$, (g) $2785 \mathrm{~ms}$, (h) $2800 \mathrm{~ms}$, (i) $3120 \mathrm{~ms}$, (j) $3145 \mathrm{~ms}$, (k) $3150 \mathrm{~ms}$, (l) $3155 \mathrm{~ms}$, (m) $3190 \mathrm{~ms}$, (n) $3205 \mathrm{~ms}$, (o) $3215 \mathrm{~ms}$, (p) $3225 \mathrm{~ms}$, (q) 3400 $\mathrm{ms}$, (r) $3405 \mathrm{~ms}$, (s) $3430 \mathrm{~ms}$, and (t) $3455 \mathrm{~ms}$.

[Figs. 5(e) and 5(f)] and the right-hand [ Figs. 5(g) and 5(h)] margins of the ischemic area, eventually propagating out of the bottom edge of the sheet. Figures 5(i)-5(1) show the propagation of the activation wave front of the eighth action potential at $\mathrm{BCL}=71 \mathrm{~ms}$. Note that the activation wave front has now definitely separated or detached from the border zone, and its free-end bears an even closer resemblance to a spiral tip. The tip then travels in the retrograde direction (i.e., right to left), eventually encountering the right-hand border zone, anchoring therein [resulting in a situation similar to that depicted in Fig. 5(h)]. As stimulation is continued, there is a progressive beat-to-beat increase in the degree of separation [Figs. $5(\mathrm{~m})-5(\mathrm{p})$ show the ninth beat), but the tip still always eventually anchors along the right-hand border of the ischemic area. Finally, the degree of separation becomes sufficiently great by the 11th beat that there is sufficient room for the spiral tip to avoid contact with the right-hand border of the ischemic area as it rotates in the clockwise direction. It thus avoids becoming anchored within the border zone, and instead rotates up into the upper-right quadrant of the sheet. A sustained spiral wave-very similar to that seen in the homogeneous sheet (Fig. 3) - is then formed, with its core lying wholly within the normal tissue in the upper-right quadrant of the sheet. Figures 5(q) -5(t) show the first turn of the spiral wave, which lasts until the end of the computation, making about ten turns. Note that there is no activation by 

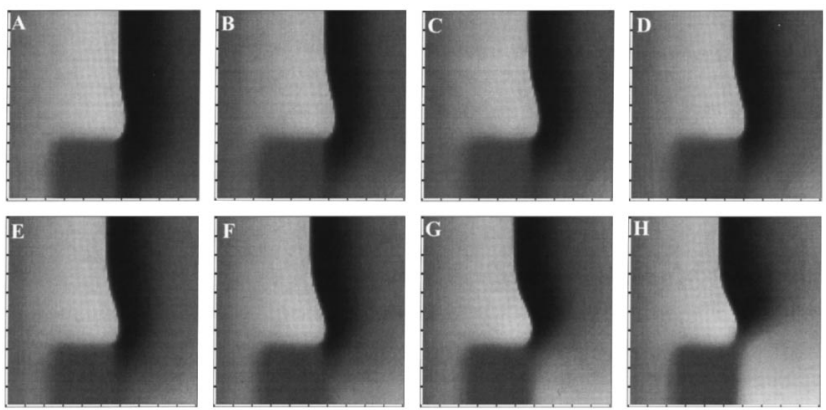

FIG. 6. Events preceding the induction of a type I spiral wave $\left(\left[\mathrm{K}^{+}\right]_{o}\right.$ $=20 \mathrm{mM}$ in the ischemic area). Note the beat-to-beat decrease in the length of the excitable gap and the beat-to-beat increase in the curvature of the activation and repolarization wave fronts. (a) The 19th action potential at $\mathrm{BCL}=74 \mathrm{~ms}$; (b) $-(\mathrm{h})$ the 5 th -11 th action potentials at $\mathrm{BCL}=71 \mathrm{~ms}$. See the text for a further discussion. Time after the start of the simulation: (a) $2485 \mathrm{~ms}$, (b) $2920 \mathrm{~ms}$, (c) $2990 \mathrm{~ms}$, (d) $3060 \mathrm{~ms}$, (e) $3130 \mathrm{~ms}$, (f) $3200 \mathrm{~ms}$, (g) $3270 \mathrm{~ms}$, and (h) $3340 \mathrm{~ms}$.

the spiral wave of the ischemic area, which remains in a state of complete block. We refer to this type of spiral wave, whose core lies within the normal area, as a type I spiral wave, to distinguish it from another form of spiral wave ("type II") that we shall encounter later.

\section{Mechanism of induction of type I spiral wave at $\left[\mathrm{K}^{+}\right]_{0}=20 \mathrm{mM}$}

Figure 6 shows frames taken at approximately equivalent times for eight different action potentials when $\left[\mathrm{K}^{+}\right]_{o}=20 \mathrm{mM}$ in the ischemic area, preceding induction of the type I spiral wave of Fig. 5. It is clear that there is a complex beat-to-beat positive feedback process, involving accumulation of refractoriness, decrease in the width of the excitable gap (both spatial and temporal), decrease in conduction velocity, and changes in the shape (especially curvature) of activation and repolarization wave fronts (most notably in the neighborhood of the upper-right-hand corner of the ischemic area). These factors all work to eventual block propagation "around the corner" of the ischemic area, but not in the upward-and-to-the-right direction. This can be appreciated quite nicely in Fig. 6(h) [Figs. 6(b)-6(h) show the last seven paced action potentials], where there is virtually no excitable gap left between the bulge of the wave front that will become the spiral tip and the repolarization wave front of the immediately preceding action potential.

\section{Type II spiral wave in an inhomogeneous sheet of muscle}

During the first few minutes of regional myocardial ischemia, there is a gradual rise in $\left[\mathrm{K}^{+}\right]_{o}$ to levels as high as $20 \mathrm{mM}^{82}$ Due to the prohibitively large computational requirements, we did not systematically explore the entire range of $\left[\mathrm{K}^{+}\right]_{o}$ encountered during ischemia $(5.4-20 \mathrm{mM})$. However, Fig. 7 shows a simulation in which $\left[\mathrm{K}^{+}\right]_{o}$ is set to $10.5 \mathrm{mM}$ in the ischemic area, and the BCL is lowered stepwise from $180 \mathrm{~ms}$ to $150,120,100,90,80,74$, and $71 \mathrm{~ms}$. At this level of $\left[\mathrm{K}^{+}\right]_{o}$, the resting potential is $-68 \mathrm{mV}$, which is less depolarized than at $\left[\mathrm{K}^{+}\right]_{o}=20 \mathrm{mM}$, so that the activation
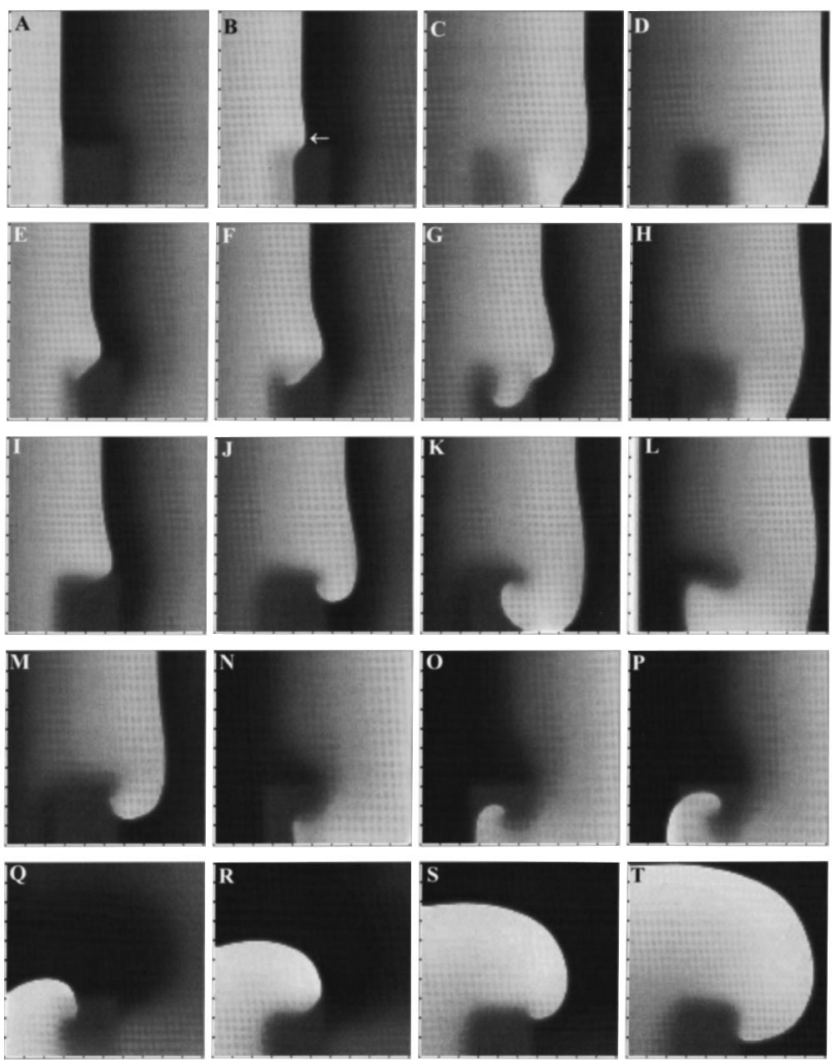

FIG. 7. A type II spiral wave in an inhomogeneous sheet $\left(\left[\mathrm{K}^{+}\right]_{o}=10.5 \mathrm{mM}\right.$ in the ischemic area). The spiral wave (type II) produced by decremental pacing protocol. See the text for a further description. Time after the start of the simulation: (a) $975 \mathrm{~ms}$, (b) $985 \mathrm{~ms}$, (c) $1010 \mathrm{~ms}$, (d) $1020 \mathrm{~ms}$, (e) 2090 $\mathrm{ms}$, (f) $2095 \mathrm{~ms}$, (g) $2105 \mathrm{~ms}$, (h) $2125 \mathrm{~ms}$, (i) $2380 \mathrm{~ms}$, (j) $2395 \mathrm{~ms}$, (k) $2405 \mathrm{~ms}$, (l) $2415 \mathrm{~ms}$, (m) $2475 \mathrm{~ms}$, (n) $2495 \mathrm{~ms}$, (o) $2505 \mathrm{~ms}$, (p) $2510 \mathrm{~ms}$, (q) $2610 \mathrm{~ms}$, (r) $2620 \mathrm{~ms}$, (s) $2630 \mathrm{~ms}$, and (t) $2640 \mathrm{~ms}$

wave front now propagates successfully through the ischemic area for all beats when the BCL is sufficiently large ( $\geqslant 80 \mathrm{~ms}$ ), although the conduction velocity increasingly slows in both the normal and the ischemic areas as BCL is decreased. Thus, for the fourth beat at $\mathrm{BCL}=80 \mathrm{~ms}$, a bulge now appears in this wave front [the arrow in Fig. 7(b)], where a free end previously appeared when $\left[\mathrm{K}^{+}\right]_{0}$ was 20 $\mathrm{mM}$ in the ischemic area [see e.g., Fig. 5(b)]. As when $\left[\mathrm{K}^{+}\right]_{o}=20 \mathrm{mM}$, the repolarization wave spreads out radially from the left-hand edge of the ischemic area, but the ischemic area itself now repolarizes first [Figs. 7(c) and 7(d)], since the APD there is shorter than in the normal area $(\sim 45$ vs. $\sim 70 \mathrm{~ms}$ ). When BCL is reduced from 80 to $74 \mathrm{~ms}$, there begins to be block of propagation through the lower part of the ischemic area: not every wave front traveling in the ischemic area penetrates all the way through to the lower righthand border of the ischemic area. There is thus "exit block" out of the lower part of the ischemic area. The normal area adjacent to the lower right-hand border is then activated by the wave front that sweeps around the upper-right corner of the ischemic area. As BCL is stepped from 74 to $71 \mathrm{~ms}$, the first action potential propagates through the ischemic area [an activation sequence similar to that shown in Figs. 7(a)7(d)], but the second and third beats show exit block. On the fourth beat, for the first time in the simulation, we now see 
entrance block: the middle of the activation wave front breaks and does not invade the central part of the left edge of the ischemic area ("partial entrance block"). On the sixth beat, there is complete entrance block into the ischemic area across its left boundary [Figs. 7(e) and 7(f)]. The lower part of the ischemic area is, however, now, for the first time, partially invaded by retrograde propagation entering from the top border [Figs. 7(f) and 7(g)]. On the tenth beat [Figs. $7(\mathrm{i})-7(1)]$ there is complete entrance block across the left and top borders of the ischemic area. The size of the retrograde activation wave front grows [Figs. 7(j) $-7(1)$ ], with increasing penetration into the ischemic area, until the point comes where the 11th and last stimulus results in a wave front that penetrates the ischemic area completely in the retrograde direction [Figs. $7(\mathrm{~m})-7(\mathrm{o})]$, emerging into the normal area adjacent to the left-hand border of the ischemic area [Fig. 7(p)]. This spiral wave makes two turns before being extinguished, with the second turn retogradely traveling through the ischemic area in a fashion similar to the first turn. Figures $7(q)-7(t)$ show the end of the second turn, which dies out after the spiral tip collides with the bottom boundary [Fig. $7(\mathrm{t})]$.

In an intact heart, each of the two nondriven beats would be identified from the electrocardiogram as a ventricular extrasystole or premature ventricular contraction (PVC), and a mapping study would record them as emerging from the ischemic area. It is well known that such premature beats can induce ventricular fibrillation, in which the normal tissue surrounding the ischemic area is involved. ${ }^{49}$ We refer to this form of spiral wave whose initiation involves retrograde propagation through the ischemic area as a type II spiral wave, to distinguish it from the type I spiral wave described earlier, which does not involve such retrograde propagation. It is possible to obtain more than two turns of the type II spiral wave (see the Discussion).

In the computations of Fig. 7 (and Fig. 8 below), the stimulus amplitude is increased to $600 \mu \mathrm{A} \mathrm{cm}^{-2}$, because the stimulus is not always able to produce a propagated response at our standard value of $300 \mu \mathrm{A} \mathrm{cm}^{-2}$.
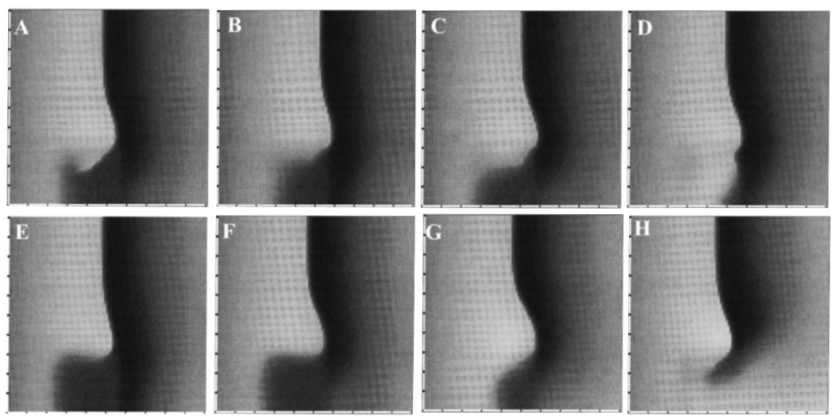

FIG. 8. Events preceding induction of a type I spiral wave $\left(\left[\mathrm{K}^{+}\right]_{o}=10.5\right.$ $\mathrm{mM}$ in the ischemic area). (a)-(h) The 6th-13th action potentials at $\mathrm{BCL}=71 \mathrm{~ms}$. See the text for a further discussion. Time after the start of the simulation: (a) $2095 \mathrm{~ms}$, (b) $2170 \mathrm{~ms}$, (c) $2240 \mathrm{~ms}$, (d) $2310 \mathrm{~ms}$, (e) 2380 $\mathrm{ms}$, (f) $2450 \mathrm{~ms}$, (g) $2520 \mathrm{~ms}$, (h) $2590 \mathrm{~ms}$.

\section{Type I spiral wave at $\left[\mathrm{K}^{+}\right]_{o}=10.5 \mathrm{mM}$}

Should additional stimuli be injected beyond the point in time at which stimulation was stopped in Fig. 7, the type II spiral wave will not be produced, since each of these additional stimuli produces an anterograde wave front that collides with the retrograde wave front resulting from the immediately preceding stimulus. Instead, with continued stimulation, a type I spiral wave eventually forms. Figure 8 shows the sequence of events leading up to the formation of the type I spiral wave at $\left[\mathrm{K}^{+}\right]_{o}=10.5 \mathrm{mM}$. Once the point in time is passed at which a type II spiral wave would start should stimulation be stopped (e.g., the wave front shown in Fig. 8(f) would form a type II spiral wave), the anterograde wave fronts traveling in the ischemic area collide with retrograde wave fronts, producing an area of complete block for the next beat. In addition, the wave front traveling in the normal area in Fig. 8(g) is blocked from traveling downward and to the right by the refractoriness left in the wake of the preceding action potential (which retrogradely invaded the ischemic area), and so is forced to travel upward and to the right [Fig. 8(h)]. The wave front shown in Fig. 8(h) thus evolves into a type I spiral wave that is similar to that seen at $\left[\mathrm{K}^{+}\right]_{o}=20 \mathrm{mM}$ [Figs. 5(q)-5(t)], except that the ischemic area, which is now no longer inexcitable at $\left[\mathrm{K}^{+}\right]_{o}$ $=10.5 \mathrm{mM}$, is activated on each turn of the spiral wave.

\section{DISCUSSION}

\section{Modifications to LR model}

The LR model has three improvements over the BR model from which it was modified: (i) a faster, more realistic, upstroke velocity due to the incorporation of a revised description of the fast inward sodium current $I_{\mathrm{Na}}$; (ii) a more accurate description of the inwardly rectifying potassium current $I_{\mathrm{K} 1}$, which involves the introduction of the plateau potassium current $I_{\mathrm{Kp}}$; (iii) the introduction of $\left[\mathrm{K}_{o}^{+}\right]$as a parameter.

We changed three parameters in the standard LR model: the time constants for activation $\left(\tau_{d}\right)$ and inactivation $\left(\tau_{f}\right)$ of the slow inward current $\left(I_{\mathrm{si}}\right)$ are divided by a factor of 12 , and the background current $\left(I_{\mathrm{b}}\right)$ is multiplied by a factor of 1.5. As detailed above, the two changes to $I_{\mathrm{si}}$ are well justified, based on the results of more recent voltage-clamp experiments on the kinetics of the L-type calcium current $I_{\mathrm{Ca}, \mathrm{L}}$. However, this modification on its own results in an APD that is too long to support a maintained spiral wave. The other currents in the LR model are $I_{\mathrm{Na}}, I_{\mathrm{K}}, I_{\mathrm{K} 1}, I_{\mathrm{Kp}}$, and $I_{\mathrm{b}}$. Since the descriptions of $I_{\mathrm{Na}}, I_{\mathrm{K}}, I_{\mathrm{K} 1}$, and $I_{\mathrm{Kp}}$ are quite plausible in the standard LR model, we decided to modify $I_{\mathrm{b}}$-which is anyway a composite current representing the hodgepodge of other currents left in the cell (e.g., Na-K pump current) once one takes into account the other currents included in the model - to reduce the APD to a workable level.

The major deficiency in our modified model is in the shape of the action potential. From infinite-rest initial conditions, the plateau phase is much depressed [trace 2 of Fig. 1(d)], starting out about $30 \mathrm{mV}$ hyperpolarized with respect to that of the unmodified LR model [trace 1 of Fig. 1(d)]. A 
triangular action potential morphology with complete loss of the plateau phase, similar to that seen in our modified model [trace 3 in Fig. 1(d)], is also seen in some experimental (e.g., Refs 27, 72) and modeling (e.g., Ref. 36) work on spiral waves. The existence of a nice plateau phase in the unmodified LR model is largely due to the fact that $I_{\text {si }}$ persists into that phase, which is, in turn, a consequence of its artefactually slow inactivation, carried over unchanged from the BR model. The other first-generation models also have a prolonged $I_{\mathrm{si}}$ phase that serves to support the plateau. The second-generation models employ either a long-lasting, sustained component of the $\mathrm{Ca}^{++}$current (termed $I_{\mathrm{Ca}, \mathrm{L}}$ or $I_{\mathrm{Ca}, \mathrm{f}}$ instead of $I_{\mathrm{si}}$ ) or the $\mathrm{Na}^{+}-\mathrm{Ca}^{++}$exchange current $I_{\mathrm{NaCa}}$ to sustain the plateau phase. ${ }^{87}$ While action-potential-clamp experiments show a long-lasting component when $I_{\mathrm{Ca}, \mathrm{L}}$ is blocked, ${ }^{88}$ the current revealed by these AP-clamp experiments is the sum of $I_{\mathrm{Ca}, \mathrm{L}}$ and any $\mathrm{Ca}^{++}$-activated currents (especially $I_{\mathrm{NaCa}}$ ). There is a pressing need for incorporating a more accurate characterization of $I_{\mathrm{NaCa}}$ and internal $\mathrm{Ca}^{++}$ handling into ionic models.

\section{Spiral waves in ionic models of homogeneous preparations}

Figure 3 shows a spiral wave in a homogeneous sheet modeled by our modified form of the LR model. A maintained spiral wave-by which we mean the persistence, without breakup, of a single spiral wave until the computation is terminated-has previously been described in homogeneous sheets of ventricular muscle using original and modified versions of four different ionic models: the Beeler and Reuter $(1977)^{50}$ model,${ }^{19,30,33,36,38}$ the Mogul et al. $(1984)^{52}$ model ${ }^{17}$ the Drouhard and Roberge (1987) ${ }^{53}$ (BRDR) model, ${ }^{34,35,42}$ and the Noble et al. $(1991)^{57}$ model. ${ }^{37,39,40} \mathrm{We}$ are not aware of any published reports using the LR model. We now go through a detailed comparison of the results of these different studies, and compare the strategies used by different authors to obtain maintained spiral-wave activity.

Unlike the case with the Noble et al. (1991) model, the BR and BRDR models have had to be modified to allow maintained spiral-wave activity. One strategy adopted (which we have also taken) in the BR and BRDR models has been to reduce the time constants $\tau_{d}$ and $\tau_{f}$ of $I_{\mathrm{si}}$, which has the effect of reducing the APD, and thus the wavelength. ${ }^{33,35,38,42}$ The same effect is achieved in the BR model by reducing $g_{\mathrm{si}}$, usually in combination with a reduction of $g_{\mathrm{Na}}$ to reduce conduction velocity. ${ }^{19,36}$ The APD of the spiral wave, which typically meanders, is reduced in these cases to $48 \pm 3-81 \pm 31 \mathrm{~ms}$ (Ref. 35); $<100 \mathrm{~ms}$ [see Fig. 1(b) of Ref. 36], and $\sim 25-200 \mathrm{~ms}$ (see Figs. 12-14 of Ref. 38). In the work using the Mogul et al. (1984) model, $\left[\mathrm{K}^{+}\right]_{o}$ is increased throughout the entire sheet to $7 \mathrm{mM}$, which reduces the wavelength since both conduction velocity and APD would presumably be reduced. ${ }^{17}$

In one study employing the BRDR model ${ }^{34}$ the bulk resistivity $[\rho$ in Eq. (1)] was $10-12 \mathrm{k} \Omega \mathrm{cm}$, which is very much higher than the nominal value of $0.2 \mathrm{k} \Omega \mathrm{cm}$ found in the literature (which is what we use here). The conduction velocity of a plane wave at $\mathrm{BCL}=500 \mathrm{~ms}$ then falls to $8.5 \mathrm{~cm} \mathrm{~s}^{-1}$, which is much lower than the value of
$35-65 \mathrm{~cm} \mathrm{~s}^{-1}$ found in the BR, BRDR, LR and Noble et al. (1991) models at a more physiological value of coupling resistance ${ }^{29,33,35,37,38,67,69,70,89}$ and in experiments on ventricular muscle. ${ }^{28,29,69,80,81}$ Hence, spiral-wave activity can be seen, even though the APD is quite long $(245 \mathrm{~ms}$ at $\mathrm{BCL}=500 \mathrm{~ms}$ ), since the low conduction velocity brings the wavelength down to only $\sim 2.1 \mathrm{~cm}$ at $\mathrm{BCL}=500 \mathrm{~ms}$. During spiral-wave activity, the APD fell to $96.7 \pm 31.3 \mathrm{~ms}$. Another consideration to be kept in mind when examining the results of this paper is that the high value of $\rho$ makes the space constant $(\lambda)$ very small, which in turn then makes the discretization factor $(\Delta x / \lambda)$ quite large..$^{90}$

In two studies where the unmodified Noble et al. (1991) model $^{57}$ has been used, ${ }^{37,39}$ the APD during spiral-wave activity is again quite small $(\sim 100$ and $\sim 120 \mathrm{~ms}$, resp.). The conduction velocity was $\sim 0.4-0.5 \mathrm{~m} \mathrm{~s}^{-1}$ for a solitary wave in the first study, while it was not reported in the second study. The APD at a long BCL is $\sim 150-200 \mathrm{~ms}$ in this guinea-pig model (see Fig. 11 of Ref. 57).

The approximate period of spiral-wave rotation reported in these modeling studies is quite variable: $\geqslant 190 \mathrm{~ms}$ (Ref. 33), $\sim 300 \mathrm{~ms}$ (Ref. 19), 200-235 ms (Ref. 38), 96.7 \pm 36.8 ms (Ref. 34), 66-117 ms (Ref. 35), 100-110 ms (Ref. 37), $\sim 100 \mathrm{~ms}$ (Ref. 39), and $70.5 \mathrm{~ms}$ (Ref. 42). The period of spiral-wave rotation in our model ( $\sim 75 \mathrm{~ms})$ thus lies toward the lower end of these results, presumably because the APD is also lower.

The elements in the spiral core are depolarized: e.g., at one site within the core there is continuous activity resembling a sine-wave oscillating between -70 and $-40 \mathrm{mV}$. Similar activity has been seen in other modeling work (e.g., Refs. 37, 91, and 92) and in experiments (e.g., Ref. 72). In contrast, in the core of some atrial rotors, one can find discrete electrotonic subthreshold events associated with block of propagation into the center of the spiral wave from its periphery (Fig. 2 of Ref. 77).

While we have focused above on ventricular muscle, spiral-wave activity has also been described in models of atrial muscle, ${ }^{65,66}$ Purkinje fiber, ${ }^{13}$ and the sinoatrial node. ${ }^{93}$

\section{Meander in ionic models of homogeneous preparations}

The tip of a spiral wave can sometimes be found to be following a closed circular or elliptical trajectory ("fixed core"). Otherwise the tip meanders. There have been two basic types of meander described in systematic studies on the FitzHugh-Nagumo (FHN) equations: simple meander and hypercomplex meander. ${ }^{32}$ In simple meander, there is a quasiperiodic motion that arises from a torus bifurcation ${ }^{94,95}$ with the tip tracing out a nice regular geometric pattern, often flower-shaped (see, e.g., Figs. 13-15 in Ref. 32). In hypermeander, the nice floral patterns are lost, and replaced by curves that are much more irregular (see e.g., Fig. 16 of Ref. 32). It has been shown that hypermeander in the FHN equations is chaotic at one particular set of parameter values. ${ }^{96}$

We know of only two instances in which fixed cores and simple meander have been unambiguously described in ventricular models: in the BR model when both $g_{\mathrm{Na}}$ and $g_{\mathrm{si}}$ are 
reduced to approximately half-normal values, ${ }^{19,36}$ and in the unmodified Noble et al. (1991) model ${ }^{39}$ (see, however, Ref. $37)$. In the BR model modified so that the kinetics of $I_{\mathrm{si}}$ are more realistic, ${ }^{33,38}$ one sees a trajectory similar to that which we find (Figs. 3 (i)-(1)), which is somewhat reminiscent of the hypercomplex meander seen in the FHN and Oregonator equations. ${ }^{32}$ The nature of the bifurcations involved in the transition from fixed core to simple meander to more complex meander in models of ventricular muscle remains to be sorted out, as does the question as to whether chaotic activity is present. Finally, it is perhaps of interest to note, in the context of our results involving type I spiral waves, that a recent study on the FHN equations shows that the transition from fixed-core motion to simple meander "is determined by the same conditions for the transition from wave-tip separation to attachment of the wave to the end of a thin ... unexcitable strip ....". 97

\section{Spiral waves in experiments on homogeneous preparations}

Based on topological considerations arising from the phase-resetting response of limit cycle oscillators, ${ }^{16,17}$ Winfree suggested that a crossed-gradient stimulation protocol should be capable of inducing spiral-wave activity in excitable cardiac tissue. This suggestion was first tested in intact dog ventricle, using an array of extracellular recording electrodes to record activation wave fronts. ${ }^{26}$ Reentrant activity (most probably scroll wave in origin), which degenerated into fibrillation, was indeed produced. The first few circuits of reentrant activity followed several of Winfree's theoretical predictions (e.g., re direction of rotation; amplitude and timing characteristics of pair of stimuli).

The activation sequence of crossed-gradient-induced reentrant activity in thin epicardial preparations has also been mapped, using either a matrix of extracellular electrodes ${ }^{28}$ or the voltage-sensitive-dye fluorescence technique..$^{27,30,72} \mathrm{In}$ addition, spiral-wave activity has been seen in epicardial sheets in which TTX was added to reduce excitability and conduction velocity, when the mechanism of initiation involves detachment from an inexcitable obstacle. ${ }^{29}$ While in the bulk of these reports it is not clear how long the spiral wave lasted, in one lab they persisted for up to an hour. ${ }^{28}$ When the spiral wave drifts and eventually anchors to an inhomogeneity (e.g., a small artery), the spiral wave persists for an indefinite period of time. ${ }^{72}$

In the most extensive report on the epicardial preparation to date ${ }^{72}$ the refractory period was $131 \mathrm{~ms}$ and the conduction velocity $0.1-0.4 \mathrm{~m} \mathrm{~s}^{-1}$ at $\mathrm{BCL}=500 \mathrm{~ms}$ (see also Table 2 of Ref. 69), thus leading to a wavelength of about $1-5 \mathrm{~cm}$. The core size was about $3 \times 6 \mathrm{~mm}$ (hemiaxial lengths), the spiral period 97-250 ms. In our modeling study the refractory period was considerably shorter $(79 \mathrm{~ms})$ and the conduction velocity at $\mathrm{BCL}=500 \mathrm{~ms}$ larger $\left(60 \mathrm{~cm} \mathrm{~s}^{-1}\right)$, leading to a wavelength $(7 \mathrm{~cm})$ that is of about the same order of magnitude as in the experimental work. Our spiral rotation period $(75 \mathrm{~ms})$ is toward the lower end of the experimentally recorded range, and thus the APD during spiral-wave reentry is considerably shorter. Our core diameter is about $5 \mathrm{~mm}$, which agrees with experiment.

\section{Spiral waves in epicardial preparations and ventricular arrhythmias}

It is not clear to us why a maintained spiral wave, albeit often drifting, ${ }^{27,72}$ can be seen in epicardial sheets, since electrical stimulation of the intact ventricle generally induces fibrillation and not monomorphic ventricular tachycardia. This is true even when the crossed-gradient protocol is used. $^{26}$ There are several possible explanations for this discrepancy. ${ }^{90,98,99}$ One possibility is that propagation in the epicardial sheets might be essentially two dimensional, and it has been recently shown in modeling work that spiral- or scroll-wave breakup, which might be the mechanism underlying the transition from tachycardia to fibrillation, is more likely in three-dimensional propagation than in twodimensional propagation, for identical cell characteristics. ${ }^{41,100,101}$ Another possibility is that the epicardial preparations are much more homogeneous than the intact heart (e.g., the apex-base gradient is removed), thus eliminating inhomogeneity-induced breakup. Drifting spiral waves have been seen in relatively homogeneous epicardial preparations, ${ }^{27,72}$ as well as in two much more heterogeneous preparations: pieces of ventricular muscle cooled to $28^{\circ} \mathrm{C}-$ $30{ }^{\circ} \mathrm{C}$, with half of each preparation treated with the drug quinidine, ${ }^{102}$ and the intact ventricle cooled to $32{ }^{\circ} \mathrm{C} .{ }^{18,20} \mathrm{It}$ has been suggested that spiral drift will result in arrhythmias resembling polymorphic ventricular tachycardia ${ }^{18,72}$ or ventricular fibrillation. ${ }^{20,21}$ It is well known that there are global gradients in the electrophysiological properties of the heart (e.g., apex-base, endocardium-epicardium) and spiral drift can be seen in simple models with a global gradient. ${ }^{86,103}$ One way out of this dilemma is to allow that ventricular fibrillation is actually the result of a single drifting spiral wave, ${ }^{20,21}$ given that most stationary spiral waves in experiments with epicardial sheets were anchored to inhomogeneities in the preparation, such as "anatomical discontinuities" 27 or "small arteries or bands of connective tissue." 72 The stability of spiral waves in the optical studies on perfused epicardial preparations might be artefactual, due to inadequate oxygenation (leading to decreased APD and conduction velocity), or pharmacological effects of the electromechanical uncoupling agent DAM (e.g., making APD smaller and the APD restitution curve less steep) (Chialvo, personal communication and Refs. 98, 99). However, the last-named eventuality does not explain spiral-wave induction in one case where DAM was not used. ${ }^{28}$ Finally, a recent study reports that out of a total of 108 episodes of atrial and ventricular tachycardia and fibrillation recorded in a variety of preparations using extracellular recording techniques, meander of one form or another was seen in only 11 instances of the cases where spiral-wave activity continued for more than two cycles. ${ }^{31}$ In addition, the spiral-wave activity was transient in these cases, producing no more than seven turns before activity was extinguished.

\section{Circus movement versus spiral wave}

The diameter of the core of the spiral wave is $\sim 5 \mathrm{~mm}$ in our model at a spiral period of $\sim 75 \mathrm{~ms}$, which is very comparable with the elliptical core size of $3.1 \times 5.5 \mathrm{~mm}$ (hemi- 
axial lengths) reported in epicardial preparations. ${ }^{72}$ The core circumference of $\sim 1.5 \mathrm{~cm}$ in our model is also very similar to the expected pathlength of a circus movement in the model, which can be estimated from the wavelength at $\mathrm{BCL}=75 \mathrm{~ms}$ to be $\sim 2 \mathrm{~cm}$ (Fig. 2). Recent experimental work in guinea-pig ventricle at $\left[\mathrm{Ca}^{++}\right]_{o}=1.25 \mathrm{mM}$ shows that circus movement reentry with a period of $97 \pm 6.2 \mathrm{~ms}$ can be produced around an anatomical obstacle $\sim 2.5 \mathrm{~cm}$ in circumference $^{80}$ (see, however, Ref. 104 for a detailed critique of this experiment). The fact that this circumference is comparable in size to that of a spiral core means that it might not be easy to distinguish circus movement from spiral-wave reentry when the grid spacing between measurement points is relatively coarse $(\sim 5-10 \mathrm{~mm})$, as is common in wholeventricle work (e.g., Ref. 105). In addition, a nice spiraling out of the activation wave fronts can be seen in experimental $^{80}$ and modeling ${ }^{92,106}$ work on circus movements. One might also adopt the point of view that a circus movement is a special form of spiral wave, since there is clearly a structure analogous to a spiral tip anchored on the circumference of the obstacle in simulations carried out on the FHN equations. ${ }^{79}$ It would therefore be incorrect to infer the absence of an anatomical obstacle simply because the activation wave fronts far from the center have a spiral shape. In fact, this observation suggests that it might be preferable to use a less ambiguous term, such as rotor, to describe what we have been calling spiral waves above.

\section{Spiral waves in models of inhomogeneous preparations}

To date, there have been far fewer articles published on modeling spiral-wave activity in an excitable medium with a localized inhomogeneity or heterogeneity than on modeling the homogeneous situation. The work on heterogeneous media in the cardiac context, starting over 30 years ago and continuing up to the present time, has almost uniformly involved individuals from the former Soviet Union. ${ }^{9-13,29,86,97,106-113}$ Balakhovsky, Krinskii, and Kholopov elegantly used the simple phenomenological concepts of conduction velocity, refractory period, and block to come to their conclusions; Petrov and Fel'd and Shcherbunov et al. used electrophysiological models; Reshetilov et al. used a Weiner-type model; and the most recent papers have employed the FHN equations in two $86,97,106,109,110,112,113$ or three ${ }^{86,111}$ spatial dimensions.

\section{Type I spiral waves in heterogeneous preparations with an impenetrable inexcitable obstacle}

When $\left[\mathrm{K}^{+}\right]_{o}=20 \mathrm{mM}$, the ischemic area is inexcitable, and one can obtain a type I spiral wave in response to fast pacing (Fig. 5). Figures 5 and 6 show that there is a very complex spatiotemporal feedback process involved in the induction of the type I spiral wave. There are three stages in obtaining a type I spiral wave: (i) the wave front must separate or detach from the obstacle so as to produce a "free end" or "wave break" ("wave front separation" or "detachment"); (ii) there must be enough space for this free end to develop into a spiral tip and turn, avoiding reattachment or anchoring to the boundary of the ischemic area; and (iii) the normal tissue outside of the obstacle must be able to support a maintained spiral wave.

Wave front separation involving an inexcitable obstacle has been produced either by fast pacing or by lowering the excitability of the medium surrounding the obstacle. In all cases reported to date, there is an extremely sharp corner at the distal end of the obstacle. The extent to which this type of geometry occurs in cardiac tissue is unclear. Perhaps the first experimental work showing spiral-wave formation via wave front separation was that carried out in the BelousovZhabotinsky (BZ) chemical reaction subjected to fast pacing. ${ }^{110,114,115}$ Similar activity was later described in thin epicardial preparations of ventricular muscle with a piece cut out so as to provide an inexcitable anatomical obstacle. ${ }^{29}$ Pacing at a very rapid rate $(\mathrm{BCL}=90-130 \mathrm{~ms}$, which is at most $10 \%$ above the BCL at which propagation failure occurred) resulted in spiral waves with a rotation period of $140 \pm 19$ ms. Modeling studies with the FHN equations reported similar phenomena. ${ }^{106,110}$

A decrease of excitability promotes separation and spiral-tip formation in the BZ reaction. ${ }^{115}$ While a decrease of excitability in the normal area around the obstacle leads to separation and spiral-wave formation in a model of another chemical reaction (the oxidation of carbon monoxide on a platinum catalyst surface), separation but not spiral-wave formation was reported in the accompanying experiments. ${ }^{116}$ In the epicardial preparation, addition of TTX, which blocks $I_{\mathrm{Na}}$ and so reduces excitability and conduction velocity, promoted separation (and the formation of a spiral wave that made at least one turn) at a much longer BCL than needed in the absence of TTX. ${ }^{29}$ While wave front separation was seen in the accompanying modeling work on the LR model when excitability was reduced by decreasing $g_{\mathrm{Na}}$, it is not clear whether a maintained spiral wave was produced. ${ }^{29}$ Earlier work in simple two-variable models of the FHN type, where the conductance of the fast inward current was reduced, showed wave front separation ${ }^{117,118}$ that led to the induction of a transient spiral wave. ${ }^{117}$ The mechanism proffered by these authors, and others working with the BZ reaction, ${ }^{115}$ is that separation occurs once the wave front curvature falls beneath the critical value that will support conduction, at which point there is a retraction or shrinking of the free end ("lateral instability" 119). More recent work on equations of the FHN type shows that rapid pacing can lead to separation and spiral-tip formation that can be "chaotic,' with the activity "resembling the initial stages of turbulence.", 120 Quantitative criteria for wave front separation from an inexcitable barrier, based on the consideration of charge balance in the FHN equations, have been derived, ${ }^{113}$ with the behavior within the "boundary layer' immediately adjacent to the obstacle being of critical importance. ${ }^{112}$

In our simulations involving a system with normal resting excitability and conduction velocity in the nonischemic area, the three major requirements to obtain wave frontseparation-induced spiral-wave formation are a sufficiently low BCL, a sufficiently large obstacle, and a sufficiently sharp corner of the obstacle.

The first major effect of fast pacing is to gradually de- 
crease excitability, thus allowing the activation wave front to detach from the obstacle. ${ }^{29,72,106,110,114,116,121}$ Two other major effects of fast pacing, reduction of APD and conduction velocity, are then also crucial in reducing the wavelength to a point that a spiral wave can form-otherwise, one might have wave front detachment alone. The obstacle must also be sufficiently large and have a sufficiently sharp corner so as to allow separation and to give the spiral tip sufficient space to turn and thus avoid reattachment to the boundary of the obstacle. ${ }^{29,106,114,115,121}$ We have not explored these two criteria systematically because of the heavy computational requirements. However, we do not see wave front separation when the angle of the upper right-hand corner of the sheet is increased from $90^{\circ}$ to $135^{\circ}$ or when the width of the obstacle (extension in the vertical direction) is reduced to $0.25 \mathrm{~cm}$. The influence of the sharpness of the corner (and of anisotropy) on the initiation of reentry is discussed in much greater depth in a recent tutorial/review article. ${ }^{104}$

\section{Type I spiral waves in heterogeneous preparations with a penetrable inexcitable obstacle}

Although the ischemic area is inexcitable in our numerical experiments at $\left[\mathrm{K}^{+}\right]_{o}=20 \mathrm{mM}$ (Figs. 5 and 6), there is still flow of current across the ischemic boundary ("penetrable" obstacle). Our simulations are thus not exactly comparable with the other works discussed immediately above, all of which use "impenetrable" obstacles with zero-flux Neumann boundary conditions. Use of impenetrable boundary conditions is only appropriate in modeling the later stages of myocardial infarction, following cell-to-cell uncoupling, cell death, and fibrosis. If one wishes to simulate the acute phase of myocardial ischemia, one must use penetrable boundary conditions.

We also carried out other simulations, setting impenetrable, rather than penetrable, boundary conditions along the edges of the ischemic area. We find that spiral-wave formation occurs at a slightly lower BCL $(75 \mathrm{~ms})$ than when penetrable boundary conditions are used with $\left[\mathrm{K}^{+}\right]_{o}=20 \mathrm{mM}$ in the ischemic area $(\mathrm{BCL}=71 \mathrm{~ms})$. We are not aware of any other simulations producing type I spiral waves using a penetrable inexcitable obstacle.

There must also be a change in the BCL at which wave front-separation-induced spiral-wave formation will occur as $\left[\mathrm{K}^{+}\right]_{o}$ in the ischemic area is systematically lowered through values below $20 \mathrm{mM}$, due to the fact that, in the limit of $\left[\mathrm{K}^{+}\right]_{o}$ in the ischemic area approaching $\left[\mathrm{K}^{+}\right]_{o}$ in the normal area, wave front separation cannot occur, since one then has a perfectly homogeneous medium.

\section{Type I spiral waves in heterogeneous preparations with an excitable obstacle}

A type I spiral wave can also be produced at $\left[\mathrm{K}^{+}\right]_{o}=10.5 \mathrm{mM}$, at which level the ischemic area is not completely inexcitable, but rather has reduced excitability (Fig. 8). Unlike the situation at $\left[\mathrm{K}^{+}\right]_{o}=20 \mathrm{mM}$, wave front separation is not involved. We are not aware of any prior modeling work showing this route to the induction of spiralwave activity. In one other study on an excitable inhomoge- neity, which used the FHN model in a three-dimensional geometry replicating the geometry of the ventricles, ${ }^{111}$ a transient spiral wave (more correctly, a scroll ring) that made three turns and was then extinguished was elicited by a $\mathrm{S}_{1}-\mathrm{S}_{2}$ protocol. This was a consequence of a wave break that occurred at the ischemic border, due to the fact that the refractory period of the ischemic area (but not that of the normal tissue) was longer than the $S_{1} S_{2}$ interval. However, we do not know if the mechanism involved there bears any resemblance to that involved in generating a type I or type II spiral wave at $\left[\mathrm{K}^{+}\right]_{o}=10.5 \mathrm{mM}$ in our study.

One fact that we find particularly intriguing about the production of a type I spiral wave at $\left[\mathrm{K}^{+}\right]_{o}=10.5 \mathrm{mM}$ is that a type II spiral wave will result should two fewer stimuli be delivered (Fig. 7). This phenomenon might be one counterpart of the well-known fact that ventricular tachycardias with different cycle times and morphologies can be provoked in patients during electrophysiological testing by changing the stimulation protocol. One other interesting report of bistability in a cardiac context involving a spiral wave is the coexistence of a spiral wave with a spiral-like circus movement around an anatomical obstacle. ${ }^{79}$

\section{Type II spiral waves in heterogeneous preparations}

A type II spiral wave (Fig. 7) by definition occurs only when the obstacle is excitable (and thus penetrable). This is exactly the condition of the ischemic area during the earliest phases of acute myocardial ischemia. Indeed it is the reduced excitability and conduction velocity leading to block within the ischemic area that is commonly implicated in the genesis of ischemic arrhythmias. ${ }^{49,105,122-124}$ Unidirectional block is clearly involved in the induction of the type II spiral wave in Fig. 7, as in the classic circus movement.

It is well known that extrasystoles or PVCs often originate either from within the ischemic area itself or from the immediately adjacent normal area. ${ }^{123}$ Our type II extrasystoles arise as a consequence of reentry within the ischemic area, with activity emerging from the border area into the normal area. As in experimental work, before extrasystoles are seen, one also obtains "continuous electrical activity", throughout the entire diastolic interval ("diastolic bridging',). ${ }^{49,125-127}$

In our simulations of type II behavior, the activation wave front must travel along the entire length of the top edge of the ischemic area and turn around the upper-right corner before it can enter the ischemic area [Fig. 7(m)]. The situation is thus similar to that described in a simple Wiener-type model, where the refractory period of the inhomogeneity is longer than that of the normal medium around it; the wave front breaks when it encounters the inhomogeneity and then keeps traveling until the inhomogeneity is out of its refractory period, whereupon the wave front enters the inhomogeneity (see Fig. 1 of Ref. 108). Similar behavior has been described during ischemia. ${ }^{49,128,129}$ Should the length of the ischemic area (extension in the horizontal direction in Fig. 4) be increased, or $\left[\mathrm{K}^{+}\right]_{o}$ lowered somewhat, or both, one might expect the wave front to enter the ischemic area across the top edge before it reaches the upper-right corner. In that 


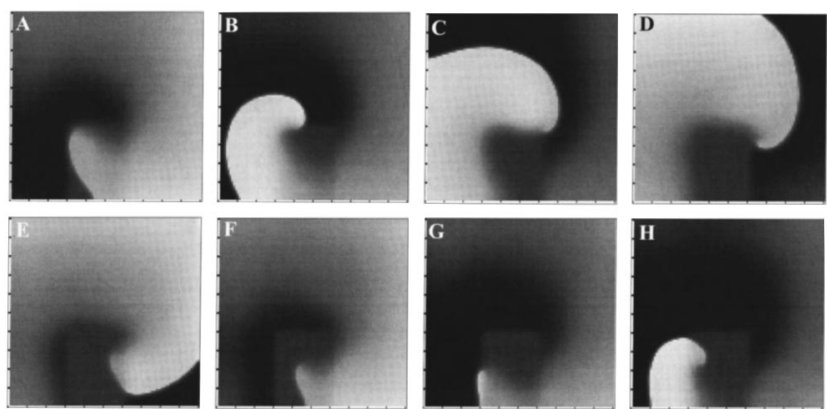

FIG. 9. A multiturn type II spiral wave $\left(\left[\mathrm{K}^{+}\right]_{o}=10.5 \mathrm{mM}\right.$ in the ischemic area). The vertical dimension of the ischemic area is increased by $0.25 \mathrm{~cm}$ so that the size of the ischemic area is now $0.75 \mathrm{~cm} \times 1.0 \mathrm{~cm}$. The second turn of the spiral wave is illustrated. The sequence of BCLs is the same as in Fig. 7.

case, a spiral wave similar to that seen in an early simulation with the FHN equations in an inhomogeneous sheet might be produced, with the spiral tip lying, at least initially, within the ischemic area (see Fig. 2 of Ref. 109).

Should the width of the ischemic area (extension in vertical direction in Fig. 4) be increased instead, and the zone placed in the center of the sheet, one can see from a symmetry argument that a pair of counter-rotating type II spiral waves might form, ${ }^{14,109}$ displaying activity resembling what is called "figure-of-eight" reentry commonly seen in experimental ischemia. ${ }^{128,129}$ In this form of reentry, there is a shared common pathway lying within the ischemic area, and the centres of the two spiral waves can lie not much farther apart than the diameter of the spiral-wave core. ${ }^{130}$ In the case of the type I spiral wave, with this geometry one would obtain two counter-rotating spiral waves initially with wellseparated cores. ${ }^{106}$

\section{Prelude to induction of type II spiral-wave activity}

The size of the ischemic area is presumably only one of the many parameters (e.g., another one being $\left[\mathrm{K}^{+}\right]_{o}$ ) that control the number of turns made by the type II spiral wave. In Fig. 7, the spiral wave was transient, dying out after only two turns. However, it is possible to obtain longer-lasting spiral-wave activity. Figure 9 shows an example in which increasing the vertical extension of the ischemic area to 1.0 $\mathrm{cm}$ from $0.75 \mathrm{~cm}$ resulted in the spiral wave making nine turns before being extinguished. It is our hypothesis that as the size of the ischemic area grows, the number of nondriven extrasystoles (PVCs) produced in response to pacing at a given rate will gradually increase until a sustained type II spiral wave is eventually formed (see also Kholopov, $1969^{12}$ ). We believe that the increase in the number of nondriven beats with an increasing size of ischemic area (or increasing $\left[\mathrm{K}^{+}\right]_{o}$ in an ischemic area) is connected with the experimental ${ }^{125}$ and clinical ${ }^{131}$ observations that, as ischemia becomes more profound, the frequency of isolated PVCs tends to increase, producing "doublets" and "triplets," until salvos of PVCs occur, followed by a run of ventricular tachycardia that rapidly degenerates into fibrillation.

Both entrance and exit block clearly occur within the ischemic area as the BCL is decreased during decremental pacing before the type II spiral wave is induced (Fig. 7). We thus expect that, for a given ischemic zone (i.e., size, placement, and level of $\left[\mathrm{K}^{+}\right]_{o}$ ), there would be a nice bifurcation sequence seen in the pattern of activation should BCL be held steady for a prolonged enough period of time at each BCL to allow the determination of the steady-state pattern of activation. For example, previous modeling work has shown that, as BCL is decreased, a direct transition to $2: 1$ block is seen in a homogeneous one-dimensional Purkinje fiber cable, and Wenckebach-like rhythms occur when the cable is made heterogeneous by inserting a central segment of diminished excitability and coupling. ${ }^{132}$ In a homogeneous onedimensional BR cable there is a direct transition to alternans and then to $2: 1$ block as BCL is decreased. ${ }^{67}$ Wenckebach $^{126-129}$ and 2:1 block, ${ }^{105,122,126}$ as well as alternans ${ }^{105,122,123,133}$ have been described in the ischemic ventricle in the prelude to induction of reentrant arrhythmias. Indeed, it has been suggested that the detection of subtle alternans on the ECG can be used as an index of vulnerability. ${ }^{134,135}$

For a type II spiral wave to be induced, one must stop pacing after a critical number of stimuli; pacing continued beyond this point results in the collision and mutual annihilation of the reentering wave front with the next stimulated wave front (Fig. 8). Analogous behavior, producing "concealed reentry," is seen in experimental work. ${ }^{129,136}$

\section{Directions for the future}

Perhaps the major weakness in our model is the rather simplistic description of the border zone and the effects of ischemia thereon. First, we have used a very sharp corner with a very acute corner angle $\left(90^{\circ}\right)$ to obtain a type I spiral wave. The arrhythmogenic potential of the type I spiral wave will depend on how sharp the corner must be in order to obtain that kind of spiral wave. Second, while we use a step discontinuity in $\left[\mathrm{K}^{+}\right]_{o}$ at the edge of the ischemic area, there is actually a gradient in $\left[\mathrm{K}^{+}\right]_{o}$ over a distance of $\sim 1 \mathrm{~cm}$ in the electrophysiologic border zone. ${ }^{82}$ Thus, in future simulations, a graded, spatially distributed border zone should be used. The decreased excitability present throughout the border zone should make wave front detachment and type I spiral-wave initiation easier at a longer BCL..$^{29,115-118}$ There has been prior work showing the initiation of a transient spiral wave in a sheet with a global gradient, ${ }^{13}$ and it has been shown that a drifting spiral wave can generate an ECG resembling fibrillation. ${ }^{20,21}$

Models used in future simulations on ischemic reentry should incorporate the $\mathrm{Na}^{+}-\mathrm{K}^{+}$pump, internal $\mathrm{Ca}^{++}$dynamics, and the $\mathrm{Na}-\mathrm{Ca}$ exchanger (as all the existing "second-generation" models mentioned above presently do) because of the well-known arrhythmogenic effect of increased intracellular $\left[\mathrm{Na}^{+}\right]$or $\left[\mathrm{Ca}^{++}\right]$. In addition, the ATPactivated $\mathrm{K}^{+}$current $I_{\mathrm{K}, \mathrm{ATP}}$ (Ref. 61) and the transient outward current $I_{\text {to }}$ (Refs. 56 and 137) should be included in the model. The known $p \mathrm{H}$ dependence of several of the currents should also be incorporated into such a model.

Our model is for the earliest part of ischemia-phase Ia (2-10 min post-occlusion) — where changes in intercellular 

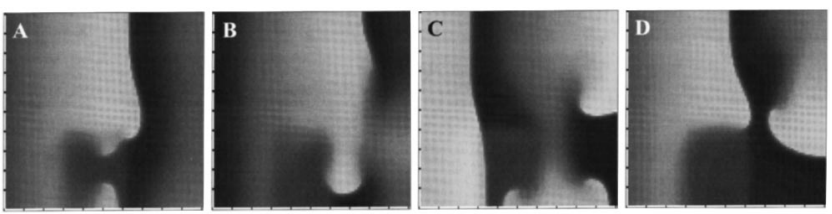

FIG. 10. Complex spatiotemporal activation pattern $\left(\left[\mathrm{K}^{+}\right]_{o}=10.5 \mathrm{mM}\right.$ in the ischemic area). Stimulation at $\mathrm{BCL}=71 \mathrm{~ms}$ is continued beyond the point in time at which first a type II, and then a type I, spiral wave would have been initiated. Ischemic area: $0.75 \mathrm{~cm} \times 1.0 \mathrm{~cm}$. Stimuli delivered to the two leftmost columns; stimulus amplitude $=300 \mu \mathrm{A} \mathrm{cm}{ }^{-2}$.

coupling do not appear to play a major role in the induction of reentry. ${ }^{138}$ Modeling of the reentrant arrhythmias occurring in the phase $\mathrm{Ib}(12-30 \text { min post-occlusion })^{138}$ and in the later phase II (several hours to several days post-infarct) ${ }^{126-128}$ stages of acute myocardial infarction will necessitate incorporation into the model of the changes in gap-junctional coupling known to take place. ${ }^{138,139}$ The slowing of conduction in this setting will facilitate the induction and maintenance of reentrant activity. Anisotropy, especially in the setting of slowed conduction, is important in phase II arrhythmogenesis, ${ }^{140}$ and so should definitely be included in any model of late arrhythmias [see, however, Winfree $(1997)^{104}$.

In our simulations, the ionic model was modified in order to lower the APD and thus the wavelength sufficiently to obtain a sustained spiral wave. Increases in computational power will permit simulations in a sheet large enough to support reentrant activity with a longer wavelength. In a model with a longer wavelength, the maintained type I spiral wave we describe here in the normal area might break up into the more complicated pattern of irregularly circulating multiple spiral waves, which might be a form of spatiotemporal chaos. ${ }^{23,141,142}$ Indeed, we have already seen very irregular activity when pacing is continued at $\left[\mathrm{K}^{+}\right]_{o}$ $=10.5 \mathrm{mM}$ beyond the point in time at which a type I spiral wave would form (Fig. 10). There is a continual production of new activation wave fronts (e.g., a blob along the right margin of the ischemic area in Fig. 10(b), which is travelling downward, is about to pinch off), with up to four wave fronts simultaneously existing on the sheet [Fig. 10(c)]. Use of a larger sheet will also allow a scaling up of the size of the ischemic area, which will then permit observation of the phenomena we describe above, but with a longer APD and a longer spiral-rotation period, both of which would be more characteristic of reentry in the canine, ${ }^{122,143}$ porcine, ${ }^{105}$ or human ${ }^{144}$ ventricle. This will then open up the door to exploring spontaneous initiation of spiral waves-which might then rapidly break up ${ }^{26}$-at a normal heart rate, which is presumably the mechanism underlying the malignant ventricular arrhythmias seen during the natural history of acute myocardial infarction.

\section{ACKNOWLEDGMENTS}

This work was supported by a grant to M. Guevara from the Medical Research Council of Canada. We wish to thank
Ali Yehia for much help with computers, and Dr. Josh Leon for providing us with time on his computer, which greatly speeded up our rate of progress.

${ }^{1}$ P. V. Bayly, B. H. KenKnight, J. M. Rogers, E. E. Johnson, R. E. Ideker, and W. M. Smith, "Spatial organization, predictability, and determinism in ventricular fibrillation," Chaos 8, 103-115 (1998).

${ }^{2}$ P.-S. Chen, A. Garfinkel, J. N. Weiss, and H. S. Karagueuzian, "Computerized mapping of fibrillation in normal ventricular myocardium," Chaos 8, 127-136 (1998).

${ }^{3}$ B. H. KenKnight, P. V. Bayly, W. M. Smith, and R. E. Ideker, "Epicardial activation patterns during regional capture of the fibrillating ventricle: Potential mechanisms and implications" (in preparation).

${ }^{4}$ F. X. Witkowski, L. J. Leon, P. A. Penkoske, R. B. Clark, M. L. Spano, W. L. Ditto, and W. R. Giles, "A method for visualization of ventricular fibrillation: Design of a cooled fiberoptically coupled image intensified CCD data acquisition system incorporating wavelet shrinkage based adaptive filtering," Chaos 8, 94-102 (1998).

${ }^{5}$ W. E. Garrey, "The nature of fibrillary contraction of the heart.-Its relation to tissue mass and form," Am. J. Physiol. 33, 397-414 (1914).

${ }^{6}$ A. G. Mayer, "Rhythmical pulsation in Scyphomedusae," Carnegie Inst. Wash. Publ. No. 47, 1906.

${ }^{7}$ G. R. Mines, "On dynamic equilibrium in the heart," J. Physiol. (London) 46, 349-383 (1913).

${ }^{8}$ G. R. Mines, "On circulating excitations in heart muscles and their possible relation to tachycardia and fibrillation," Trans. R. Soc. Can. Ser. 3, Sect. IV 8, 43-52 (1914).

${ }^{9}$ I. S. Balakhovskii, "Several modes of excitation movement in ideal excitable tissue," Biophysics 10, 1175-1179 (1965).

${ }^{10}$ V. I. Krinskii, "Spread of excitation in an inhomogeneous medium (state similar to cardiac fibrillation)," Biophysics 11, 776-784 (1966).

${ }^{11}$ A. V. Kholopov, "Spread of excitation and the formation of closed pathways of conduction in a plane medium the refractoriness of which depends on the period of excitation," Biophysics 13, 1231-1243 (1967).

${ }^{12}$ A. V. Kholopov, "Effect of the parameters and properties of an excitable medium on the process of fibrillation," Biophysics 14, 726-734 (1969).

${ }^{13}$ A. I. Shcherbunov, N. I. Kukushkin, and M. Y. Sakson, "Reverberator in a system of interrelated fibres described by the Noble equation," Biophysics 18, 547-554 (1973).

${ }^{14}$ A. M. Pertsov and A. K. Grenadier, "The autowave nature of cardiac arrhythmias," in Self-Organization: Autowaves and Structures far from Equilibrium, edited by V. I. Krinsky (Springer-Verlag, Berlin, 1984), pp. 184-190.

${ }^{15}$ A. T. Winfree, When Time Breaks Down. The Three-Dimensional Dynamics of Electrochemical Waves and Cardiac Arrhythmias (Princeton University Press, Princeton, 1987).

${ }^{16}$ A. T. Winfree, "Sudden cardiac death: A problem in topology," Sci. Am. 248(5), 144-161 (1983).

${ }^{17}$ A. T. Winfree, "Electrical instability in cardiac muscle: Phase singularities and rotors," J. Theor. Biol. 138, 353-405 (1989).

${ }^{18}$ R. A. Gray, J. Jalife, A. Panfilov, W. T. Baxter, C. Cabo, J. M. Davidenko, and A. M. Pertsov, "Nonstationary vortexlike reentrant activity as a mechanism of polymorphic ventricular tachycardia in the isolated rabbit heart," Circulation 91, 2454-2469 (1995).

${ }^{19}$ C. F. Starmer, D. N. Romashko, R. S. Reddy, Y. I. Zilberter, J. Starobin, A. O. Grant, and V. I. Krinsky, "Proarrhythmic response to potassium channel blockade. Numerical studies of polymorphic tachyarrhythmias," Circulation 92, 595-605 (1995).

${ }^{20}$ R. A. Gray, J. Jalife, A. V. Panfilov, W. T. Baxter, C. Cabo, J. M. Davidenko, and A. M. Pertsov, "Mechanisms of cardiac fibrillation," Science 270, 1222-1223 (1995).

${ }^{21}$ J. Jalife, R. A. Gray, G. E. Morley, and J. M. Davidenko, "Selforganization and the dynamical nature of ventricular fibrillation," Chaos 8, 79-93 (1998).

${ }^{22}$ G. K. Moe, W. C. Rheinboldt, and J. A. Abildskov, "A computer model of atrial fibrillation," Am. Heart J. 67, 200-221 (1964).

${ }^{23}$ A. V. Panfilov and A. V. Holden, "Spatiotemporal irregularity in a twodimensional model of cardiac tissue," Int. J. Bifurcation Chaos Appl. Sci. Eng. 1, 219-225 (1991).

${ }^{24}$ A. V. Panfilov, "Spiral breakup as a model of ventricular fibrillation," Chaos 8, 57-64 (1998).

${ }^{25}$ M. A. Allessie, F. I. M. Bonke, and F. J. G. Schopman, "Circus move- 
ment in rabbit atrial muscle as a mechanism of tachycardia," Circ. Res. 33, 54-62 (1973).

${ }^{26}$ D. W. Frazier, P. D. Wolf, J. M. Wharton, A. S. L. Tang, W. M. Smith, and R. E. Ideker, "Stimulus-induced critical point: Mechanism for electrical initiation of reentry in normal canine myocardium,' J. Clin. Invest. 83, 1039-1052 (1989).

${ }^{27}$ J. M. Davidenko, A. V. Pertsov, R. Salomonsz, W. Baxter, and J. Jalife, "Stationary and drifting spiral waves of excitation in isolated cardiac muscle," Nature (London) 355, 349-351 (1992).

${ }^{28}$ C. A. Nour, J. L. Leon, M. Vermeulen, R. Cardinal, and F. A. Roberge, "Mapping of reentry in a thin sheet of canine myocardium," 17th EMBS Conference (IEEE, Montreal, 1995), Paper 1.1.6.6.

${ }^{29}$ C. Cabo, A. M. Pertsov, J. M. Davidenko, W. T. Baxter, R. A. Gray, and J. Jalife, "Vortex shedding as a precursor of turbulent electrical activity in cardiac muscle," Biophys. J. 70, 1105-1111 (1996).

${ }^{30}$ N. V. Thakor and M. G. Fishler, "Initiation and termination of spiral waves in a two-dimensional bidomain model of cardiac tissue," Computers in Cardiology (IEEE Computer Society, Silver Springs, 1996), pp. 229-232.

${ }^{31}$ D. T. Kim, Y. Kwan, J. J. Lee, T. Ikeda, T. Uchida, K. Kamjoo, Y.-H. Kim, J. J. C. Ong, C. A. Athill, T.-J. Wu et al., "Patterns of spiral tip motion in cardiac tissues," Chaos 8, 137-148 (1998).

${ }^{32}$ A. T. Winfree, "Varieties of spiral wave behavior: An experimentalist's approach to the theory of excitable media,' Chaos 1, 303-334 (1991).

${ }^{33} \mathrm{M}$. Courtemanche and A. T. Winfree, "Re-entrant rotating waves in a Beeler-Reuter based model of two-dimensional cardiac electrical activity," Int. J. Bifurcation Chaos Appl. Sci. Eng. 1, 431-444 (1991).

${ }^{34}$ M. G. Fishler and N. V. Thakor, "A massively parallel computer model of propagation through a two-dimensional cardiac syncytium," PACE 14, 1694-1699 (1991)

${ }^{35}$ L. J. Leon, F. A. Roberge, and A. Vinet, "Simulation of two-dimensional anisotropic cardiac reentry: effects of the wavelength on the reentry characteristics,"' Ann. Biomed. Eng. 22, 592-609 (1994).

${ }^{36}$ I. R. Efimov, V. I. Krinsky, and J. Jalife, "Dynamics of rotating vortices in the Beeler-Reuter model of cardiac tissue,' Chaos Solitons Fractals 5, 513-526 (1995).

${ }^{37}$ V. N. Biktashev and A. V. Holden, "Re-entrant activity and its control in a model of mammalian ventricular tissue," Proc. R. Soc. London, Ser. B 263, 1373-1382 (1996)

${ }^{38} \mathrm{M}$. Courtemanche, "Complex spiral wave dynamics in a spatially distributed ionic model of cardiac electrical activity," Chaos 6, 579-600 (1996).

${ }^{39}$ L. A. Irvine and R. L. Winslow, "Numerical studies of use-dependent block of cardiac sodium channels by quinidine on spiral wave reentry," in Ref. 30, pp. 613-616.

${ }^{40}$ V. N. Biktashev and A. V. Holden, "Re-entrant waves and their elimination in a model of mammalian ventricular tissue," Chaos 8, 48-56 (1998).

${ }^{41} \mathrm{~F}$. Fenton and A. Karma, "Vortex dynamics in three-dimensional continuous myocardium with fiber rotation: Filament instability and fibrillation," Chaos 8, 20-47 (1998).

${ }^{42}$ N. Trayanova, K. Scouibine, and F. Aguel, "The role of cardiac tissue structure in defibrillation," Chaos 8, 221-233 (1998).

${ }^{43}$ T. Watanabe, P. M. Rautaharju, and T. F. McDonald, "Ventricular action potentials, ventricular extracellular potentials, and the ECG of guinea pig,"' Circ. Res. 57, 362-373 (1985).

${ }^{44}$ D. S. Rosenbaum, D. T. Kaplan, A. Kanai, L. Jackson, H. Garan, R. J. Cohen, and G. Salama, "Repolarization inhomogeneities in ventricular myocardium change dynamically with abrupt cycle length shortening," Circulation 84, 1333-1345 (1991).

${ }^{45}$ C. Antzelevitch, S. Sicouri, S. H. Litovsky, A. Lukas, S. C. Krishnan, J. M. Di Diego, G. A. Gintant, and D.-W. Liu, "Heterogeneity within the ventricular wall: Electrophysiology and pharmacology of epicardial, endocardial, and M cells," Circ. Res. 69, 1427-1449 (1991).

${ }^{46}$ J. Han and G. K. Moe, "Nonuniform recovery of excitability in ventricular muscle," Circ. Res. 14, 44-60 (1964).

${ }^{47}$ M. J. Janse, "Vulnerability to ventricular fibrillation," Chaos (in press).

${ }^{48}$ C.-S. Kuo, K. Munakata, C. P. Reddy, and B. Surawicz, "Characteristics and possible mechanism of ventricular arrhythmia dependent on the dispersion of action potential durations," Circulation 67, 1356-1367 (1983).

${ }^{49}$ M. J. Janse and A. L. Wit, "Electrophysiological mechanisms of ventricular arrhythmias resulting from myocardial ischemia and infarction," Physiol. Rev. 69, 1049-1169 (1989).

${ }^{50}$ G. W. Beeler and H. Reuter, "Reconstruction of the action potential of ventricular myocardial fibres,"' J. Physiol. (London) 268, 177-210 (1977).

${ }^{51}$ J.-P. Drouhard and F. A. Roberge, "A simulation study of the ventricular myocardial action potential," IEEE Trans. Biomed. Eng. BME-29, 494502 (1982).

${ }^{52}$ D. J. Mogul, N. V. Thakor, J. R. McCullough, G. A. Myers, R. E. ten Eick, and D. H. Singer, "Modified Beeler-Reuter model yields improved simulation of myocardial action potentials," Computers in Cardiology, (IEEE Computer Society, Silver Springs, 1984), pp. 159-162.

${ }^{53}$ J.-P. Drouhard and F. A. Roberge, "Revised formulation of the HodgkinHuxley representation of the sodium current in cardiac cells," Comput. Biomed. Res. 20, 333-350 (1987).

${ }^{54}$ D. R. Lemieux, F. A. Roberge, and P. Savard, "A model study of the contribution of active $\mathrm{Na}-\mathrm{K}$ transport to membrane repolarization in cardiac cells," J. Theor. Biol. 142, 1-33 (1990).

${ }^{55} \mathrm{C}$. Luo and Y. Rudy, "A model of the ventricular cardiac action potential," Circ. Res. 68, 1501-1526 (1991).

${ }^{56}$ A. V. Sahakian, G. A. Mayers, and N. Maglaveras, "Unidirectional block in cardiac fibers: Effects of discontinuities in coupling resistance and spatial changes in resting membrane potential in a computer simulation study,"' IEEE Trans. Biomed. Eng. 39, 510-521 (1992).

${ }^{57}$ D. Noble, S. J. Noble, G. C. L. Bett, Y. E. Earm, W. K. Ho, and I. K. So, "The role of sodium-calcium exchange during the cardiac action potential," Ann. NY Acad. Sci. 639, 334-353 (1991).

${ }^{58} \mathrm{C}$. Nordin, "Computer model of membrane current and intracellular $\mathrm{Ca}^{++}$ flux in the isolated guinea pig ventricular myocyte," Am. J. Physiol. 265, H2117-H2136 (1993).

${ }^{59} \mathrm{C}$. H. Luo and Y. Rudy, "A dynamic model of the cardiac ventricular action potential," Circ. Res. 74, 1071-1096 (1994).

${ }^{60}$ J. Zeng, K. R. Laurita, D. S. Rosenbaum, and Y. Rudy, “"Two components of the delayed rectifier $\mathrm{K}^{+}$current in ventricular myocytes of the guinea pig type. Theoretical formulation and their role in repolarization," Circ. Res. 77, 140-152 (1995).

${ }^{61}$ J. M. Ferrero, Jr., J. Saiz, J. M. Ferrero, and N. V. Thakor, “'Simulation of action potentials from metabolically impaired cardiac myocytes: role of ATP-sensitive $\mathrm{K}^{+}$current,' Circ. Res. 79, 208-221 (1996).

${ }^{62}$ N. Friedman, A. Vinet, and F. A. Roberge, "A study of a new model of the cardiac ventricular cell incorporating myoplasmic calcium regulation," Proceedings of the 22nd CMBES Conference (IEEE, Montreal, 1996).

${ }^{63}$ S. Dokos, B. G. Celler, and N. H. Lovell, "Modification of DiFrancescoNoble equations to simulate the effects of vagal stimulation on in vivo mammalian sinoatrial node electrical activity," Ann. Biomed. Eng. 21, 321-335 (1993).

${ }^{64}$ Y. E. Earm and D. Noble, "A model of the single atrial cell: Relation between calcium current and calcium release," Proc. R. Soc. London, Ser. B 240, 83-96 (1990).

${ }^{65} \mathrm{~V}$. N. Biktashev and A. V. Holden, "Control of re-entrant activity in a model of mammalian atrial tissue," Proc. R. Soc. London, Ser. B 260, 211-217 (1995)

${ }^{66} \mathrm{~A}$. V. Holden and H. Zhang, "Characteristics of atrial re-entry and meander computed from a model of a rabbit single atrial cell," J. Theor. Biol. 175, 545-551 (1995).

${ }^{67}$ T. J. Lewis and M. R. Guevara, "Chaotic dynamics in an ionic model of the propagated cardiac action potential," J. Theor. Biol. 146, 407-432 (1990).

${ }^{68} \mathrm{H}$. Windisch, W. Müller, and H. A. Tritthart, "Fluorescence monitoring of rapid changes in membrane potential in heart muscle,' Biophys. J. 48, 877-884 (1985).

${ }^{69}$ C. Cabo, A. M. Pertsov, W. T. Baxter, J. M. Davidenko, R. A. Gray, and J. Jalife, "Wave-front curvature as a cause of slow conduction and block in isolated cardiac muscle," Circ. Res. 75, 1014-1028 (1994).

${ }^{70}$ V. G. Fast and A. G. Kléber, "Block of impulse propagation at an abrupt tissue expansion: Evaluation of the critical strand diameter in 2- and 3dimensional computer models," Cardiovasc. Res. 30, 449-459 (1995).

${ }^{71}$ L. Lapidus and G. F. Pinder, Numerical Solution of Partial Differential Equations in Science and Engineering (Wiley, New York, 1982).

${ }^{72}$ A. M. Pertsov, J. M. Davidenko, R. Salomonsz, W. T. Baxter, and J. Jalife, "Spiral waves of excitation underlie reentrant activity in isolated cardiac muscle," Circ. Res. 72, 631-650 (1993).

${ }^{73} \mathrm{G}$. Isenberg and U. Klöckner, "Calcium currents of isolated bovine ventricular myocytes are fast and of large amplitude," Pflügers Arch. Ges. Physiol. Menschen Tiere 395, 30-41 (1982).

${ }^{74} \mathrm{D}$. Noble, "The surprising heart: A review of recent progress in cardiac electrophysiology,"' J. Physiol. (London) 353, 1-50 (1984).

${ }^{75} \mathrm{G}$. Isenberg and U. Klöckner, "Isolated bovine ventricular myocytes: 
Characterization of the action potential," Pflügers Arch. Ges. Physiol. Menschen Tiere 395, 19-29 (1982).

${ }^{76}$ A. Varró, D. A. Lathrop, S. B. Hester, P. P. Nánási, and J. G. Papp, “'Ionic currents and action potentials in rabbit, rat, guinea pig ventricular myocytes," Bas. Res. Cardiol. 88, 93-102 (1993).

${ }^{77}$ M. A. Allessie, F. I. M. Bonke, and F. J. G. Schopman, "Circus movement in rabbit atrial muscle as a mechanism of tachycardia III. The "leading circle' concept: A new model of circus movement in cardiac tissue without the involvement of an anatomical obstacle,' Circ. Res. 41, 9-18 (1977).

${ }^{78}$ J. L. R. M. Smeets, M. A. Allessie, W. J. E. P. Lammers, F. I. M. Bonke, and J. Hollen, "The wavelength of the cardiac impulse and reentrant arrhythmias in isolated rabbit atrium: The role of heart rate, autonomic transmitters, temperature, and potassium," Circ. Res. 58, 96-108 (1986).

${ }^{79}$ A. M. Pertsov, E. A. Ermakova, and A. V. Panfilov, "Rotating spiral waves in a modified Fitz-Hugh-Nagumo model,', Physica D 14, 117-124 (1984).

${ }^{80}$ S. D. Girouard, J. M. Pastore, K. R. Laurita, K. W. Gregory, and D. S. Rosenbaum, "Optical mapping in a new guinea pig model of ventricular tachycardia reveals mechanisms for multiple wavelengths in a single reentrant circuit," Circulation 93, 603-613 (1996).

${ }^{81}$ M. J. Schalij, “Anisotropic conduction and ventricular tachycardia," Doctoral thesis, University of Limburg, Maastricht, 1988.

${ }^{82}$ R. Coronel, J. W. Fiolet, F. J. G. Wilms-Schopman, A. F. Schaapherder, T. A. Johnson, L. S. Gettes, and M. J. Janse, "Distribution of extracellular potassium and its relation to electrophysiologic changes during acute myocardial ischemia in the isolated perfused porcine heart," Circulation 77, 1125-1138 (1988).

${ }^{83}$ R. Coronel, J. W. Fiolet, J. G. Wilms-Schopman, T. Opthof, A. F. Schaapherder, and M. J. Janse, "Distribution of extracellular potassium and electrophysiologic changes during two-stage coronary ligation in the isolated, perfused canine heart," Circulation 80, 165-177 (1989).

${ }^{84}$ R. Coronel, F. J. G. Wilms-Schopman, T. Opthof, J. Cinca, J. W. Fiolet, and M. J. Janse, "Reperfusion arrhythmias in isolated perfused pig hearts. Inhomogeneities in extracellular potassium, ST and TQ potentials, and transmembrane action potentials," Circ. Res. 71, 1131-1142 (1992).

${ }^{85}$ R. Coronel, F. J. G. Wilms-Schopman, J. W. Fiolet, T. Opthof, and M. J. Janse, "The relation between extracellular potassium concentration and $\mathrm{pH}$ in the border zone during regional ischemia in isolated porcine hearts," J. Mol. Cell. Cardiol. 27, 2069-2073 (1995).

${ }^{86}$ M. Vinson, A. M. Pertsov, and J. Jalife, "Anchoring of vortex filaments in 3D excitable media," Physica D 72, 119-134 (1993).

${ }^{87} \mathrm{~N}$. C. Janvier and M. R. Boyett, "The role of Na-Ca exchange current in the cardiac action potential," Cardiovasc. Res. 32, 69-84 (1996).

${ }^{88}$ T. Doerr, R. Denger, A. Doerr, and W. Trautwein, "Ionic currents contributing to the action potential in single ventricular myocytes of the guinea-pig studied with action potential clamp," Pflügers Arch. Ges. Physiol. Menschen Tiere 416, 230-237 (1990).

${ }^{89}$ G. H. Sharp and R. W. Joyner, "Simulated propagation of cardiac action potentials," Biophys. J. 31, 403-423 (1980).

${ }^{90}$ A. T. Winfree. "How does ventricular tachycardia decay into ventricular fibrillation?," in Cardiac Mapping, edited by M. Shenasa, M. Borggrefe, and G. Breithardt (Futura, New York, 1993), pp. 655-682.

${ }^{91}$ F. J. L. van Capelle and D. Durrer, "Computer simulation of arrhythmias in a network of coupled excitable elements," Circ. Res. 47, 454-466 (1980).

${ }^{92}$ F. J. L. van Capelle and M. A. Allessie, "Computer simulation of anisotropic impulse propagation: Characteristics of action potentials during reentrant arrhythmias," in Cell to Cell Signalling: From Experiments to Theoretical Models, edited by A. Goldbeter (Academic, London, 1989), pp. 577-588

${ }^{93}$ M. A. Mercader, D. C. Michaels, and J. Jalife, "Reentrant activity in the form of spiral waves in mathematical models of the sinoatrial node," in Cardiac Electrophysiology: From Cell to Bedside, edited by D. P. Zipes and J. Jalife, 2nd ed. (Saunders, Philadelphia, 1995), pp. 389-403.

${ }^{94}$ D. Barkley, M. Kness, and L. S. Tuckerman, "Spiral-wave dynamics in a simple model of excitable media: The transition from simple to compound rotation," Phys. Rev. A 42, 2489-2492 (1990).

${ }^{95}$ D. Barkley and I. G. Kevrekidis, “'A dynamical systems approach to spiral wave dynamics," Chaos 4, 453-460 (1994).

${ }^{96} \mathrm{H}$. Zhang and A. V. Holden, "Chaotic meander of spiral waves in the FitzHugh-Nagumo system,' Chaos Solitons Fractals 5, 661-670 (1995).

${ }^{97}$ J. M. Starobin and C. F. Starmer, "Common mechanism links spiral wave meandering and wave-front-obstacle separation," Phys. Rev. E 55, 1193-1196 (1997).

${ }^{98}$ A. T. Winfree, "Electrical turbulence in three-dimensional heart muscle," Science 266, 1003-1006 (1994).

${ }^{99}$ A. T. Winfree, "Rotors, fibrillation and dimensionality," in Computational Biology of the Heart, edited by A. V. Panfilov and A. V. Holden (J. Wiley, Chichester, 1997), pp. 101-135.

${ }^{100}$ A. Panfilov and P. Hogeweg, "Spiral breakup in a modified FitzHughNagumo model,'” Phys. Lett. A 176, 295-299 (1993).

${ }^{101}$ A. V. Panfilov and P. Hogeweg, "Scroll breakup in a three-dimensional excitable medium," Phys. Rev. E 53, 1740-1743 (1996).

${ }^{102}$ V. G. Fast and A. M. Pertsov, "Drift of a vortex in the myocardium," Biophysics 35, 489-494 (1990).

${ }^{103}$ A. M. Pertsov and Y. A. Yermakova, "Mechanism of drift of a helical wave in an inhomogeneous medium," Biophysics 33, 364-369 (1988).

${ }^{104}$ A. T. Winfree, "Heart muscle as a reaction-diffusion medium: The roles of electric potential diffusion, activation front curvature, and anisotropy," Int. J. Bifurcation Chaos Appl. Sci. Eng. 7, 487-526 (1997).

${ }^{105}$ E. Downar, M. J. Janse, and D. Durrer, "The effect of acute coronary artery occlusion on subepicardial transmembrane potentials in the intact porcine heart," Circulation 56, 217-224 (1977).

${ }^{106}$ A. V. Panfilov and J. P. Keener, "Effects of high frequency stimulation on cardiac tissue with an inexcitable obstacle,"' J. Theor. Biol. 163, 439448 (1993)

${ }^{107}$ A. A. Petrov and B. N. Fel'd, "Analysis of the possible mechanism of origin of the extrasystole in local ischaemia of the myocardium using a mathematical model," Biophysics 18, 1145-1150 (1973).

${ }^{108}$ A. N. Reshetilov, A. M. Pertsov, and V. I. Krinskii, "Parameters of the myocardium controlling vulnerability. Analysis of mathematical models," Biophysics 24, 133-139 (1979).

${ }^{109}$ A. V. Panfilov and B. N. Vasiev, "Vortex initiation in a heterogeneous excitable medium," Physica D 49, 107-113 (1991).

${ }^{110}$ K. I. Agladze, "High-frequency instability of wave fronts," Chaos 4 , 525-529 (1994).

${ }^{111}$ R. R. Aliev and A. V. Panfilov, "Modeling of heart excitation patterns caused by a local inhomogeneity," J. Theor. Biol. 181, 33-40 (1996).

${ }^{112}$ J. M. Starobin and C. F. Starmer, "Boundary-layer analysis of waves propagating in an excitable medium: Medium conditions for wave-frontobstacle separation,' Phys. Rev. E 54, 430-437 (1996).

${ }^{113}$ J. M. Starobin, Y. I. Zilberter, E. M. Rusnak, and C. F. Starmer, "Wavelet formation in excitable cardiac tissue: The role of wavefront-obstacle interactions in initiating high-frequency fibrillatory-like arrhythmias," Biophys. J. 70, 581-594 (1996).

${ }^{114}$ K. Agladze, J. P. Keener, S. C. Müller, and A. Panfilov, "Rotating spiral waves created by geometry," Science 264, 1746-1748 (1994).

${ }^{115}$ M. Gómez-Gesteira, J. L. del Castillo, M. E. Vázquez-Iglesias, V. PérezMuñuzuri, and V. Pérez-Villar, "Influence of the critical curvature on spiral initiation in an excitable medium," Phys. Rev. E 50, 4646-4649 (1994).

${ }^{116}$ M. Bär, A. K. Bangia, I. G. Kevrekidis, G. Haas, H.-H. Rotermund, and G. Ertl, "Composite catalyst surfaces: Effect of inert and active heterogeneities on pattern formation," J. Phys. Chem. 100, 19 106-19 117 (1996).

${ }^{117}$ A. V. Panfilov and A. M. Pertsov, "Mechanism of the origin of the helical waves in active media associated with the phenomenon of critical curvature," Biophysics 27, 931-934 (1982).

${ }^{118}$ A. M. Pertsov, A. V. Panfilov, and F. U. Medvedeva, "Instability of autowaves in excitable media associated with the phenomenon of critical curvature," Biophysics 28, 103-107 (1983).

${ }^{119}$ Z. Nagy-Ungvarai, A. M. Pertsov, B. Hess, and S. C. Müller, "Lateral instabilities of a wave front in the Ce-catalyzed Belousov-Zhabotinsky reaction," Physica D 61, 205-212 (1992).

${ }^{120}$ A. M. Pertsov, E. A. Ermakova, and E. E. Shnol, "'On the diffraction of autowaves,'” Physica D 44, 178-190 (1990).

${ }^{121}$ A. V. Panfilov and J. P. Keener, "Generation of reentry in anisotropic myocardium,” J. Cardiovasc. Electrophysiol. 4, 412-421 (1993).

${ }^{122}$ D. C. Russell, H. J. Smith, and M. F. Oliver, "Transmembrane potential changes and ventricular fibrillation during repetitive myocardial ischaemia in the dog,"' Br. Heart J. 42, 88-96 (1979).

${ }^{123}$ M. J. Janse, F. J. L. van Capelle, H. Morsink, A. G. Kléber, F. WilmsSchopman, R. Cardinal, C. Naumann D'Alnoncourt, and D. Durrer, ' Flow of 'injury' current and patterns of excitation during early ventricular arrhythmias in acute regional myocardial ischemia in isolated porcine 
and canine hearts. Evidence for two different arrhythmogenic mechanisms," Circ. Res. 47, 151-165 (1980).

${ }^{124}$ R. Coronel, "Distribution of extracellular potassium during acute myocardial ischemia," Doctoral thesis, University of Amsterdam, Amsterdam, 1988.

${ }^{125}$ A. S. Harris and A. Guevara Rojas, "The initiation of ventricular fibrillation due to coronary occlusion,' Exp. Med. Surg. 1, 105-122 (1943).

${ }^{126}$ N. El-Sherif, B. J. Scherlag, R. Lazzara, and R. R. Hope, "Re-entrant ventricular arrhythmias in the late myocardial infarction period: 1 . Conduction characteristics in the infarction zone," Circulation 55, 686-702 (1977).

${ }^{127}$ N. El-Sherif, R. R. Hope, B. J. Scherlag, and R. Lazzara, "Re-entrant ventricular arrhythmias in the late myocardial infarction period. 2. Patterns of initiation and termination of reentry," Circulation 55, 702-719 (1977).

${ }^{128}$ N. El-Sherif, W. B. Gough, R. H. Zeiler, and R. Hariman, "Reentrant ventricular arrhythmias in the late myocardial infarction period. 12 . Spontaneous versus induced reentry and intramural versus epicardial circuits," J. Am. Coll. Cardiol. 6, 124-132 (1985).

${ }^{129}$ M. A. Habbab and N. El-Sherif, "Recordings from the slow zone of reentry during burst pacing versus programmed premature stimulation for initiation of reentrant ventricular tachycardia in patients with coronary artery disease,', Am. J. Cardiol. 70, 211-217 (1992).

${ }^{130}$ E. A. Ermakova, A. M. Pertsov, and E. E. Shnol, "On the interaction of vortices in two-dimensional active media," Physica D 40, 185-195 (1989).

${ }^{131}$ L. E. Hinkle, Jr., D. C. Argyros, J. C. Hayes, T. Robinson, D. R. Alonso, S. C. Shipman, and M. E. Edwards, "Pathogenesis of an unexpected sudden death: Role of early cycle ventricular premature contractions," Am. J. Cardiol. 39, 873-879 (1977).

${ }^{132}$ M. R. Guevara, "Spatiotemporal patterns of block in an ionic model of cardiac Purkinje fibre," in From Chemical to Biological Organization, edited by M. Markus, S. C. Müller, and G. Nicolis (Springer-Verlag, Berlin, 1988), pp. 273-281.

${ }^{133}$ H. Moréna, M. J. Janse, J. W. T. Fiolet, W. J. G. Krieger, H. Crijns, and D. Durrer, "Comparison of the effects of regional ischemia, hypoxia, hyperkalemia, and acidosis on intracellular and extracellular potentials and metabolism in the isolated porcine heart," Circ. Res. 46, 634-646 (1980).
${ }^{134}$ B. D. Nearing, A. H. Huang, and R. L. Verrier, "Dynamic tracking of cardiac vulnerability by complex demodulation of the T wave," Science 252, 437-440 (1991)

${ }^{135}$ D. S. Rosenbaum, L. E. Jackson, J. M. Smith, H. Garan, J. N. Ruskin, and R. J. Cohen, "Electrical alternans and vulnerability to ventricular arrhythmias," New England J. Med. 330, 235-241 (1994).

${ }^{136}$ N. El-Sherif, R. Mehra, W. B. Gough, and R. H. Zeiler, "Reentrant ventricular arrhythmias in the late myocardial infarction period. II. Burst pacing versus multiple premature stimulation in the induction of reentry," J. Am. Coll. Cardiol. 4, 295-304 (1984).

${ }^{137}$ A. Ogbaghebriel and A. Shrier, "Inhibition of metabolism abolishes transient outward current in rabbit atrial myocytes," Am. J. Physiol. 266, H182-H190 (1994).

${ }^{138}$ W. T. Smith IV, W. F. Fleet, T. A. Johnson, C. L. Engle, and W. E. Cascio, "The Ib phase of ventricular arrhythmias in ischemic in situ porcine heart is related to changes in cell-to-cell electrical coupling," Circulation 92, 3051-3060 (1995).

${ }^{139}$ J. T. Vermeulen, H. L. Tan, H. Rademaker, C. A. Schumacher, P. Loh, T. Opthof, R. Coronel, and M. J. Janse, "Electrophysiologic and extracellular ionic changes during acute ischemia in failing and normal rabbit myocardium,"' J. Mol. Cell. Cardiol. 28, 123-131 (1996).

${ }^{140}$ S. M. Dillon, M. A. Allessie, P. C. Ursell, and A. L. Wit, "Influences of anisotropic tissue structure on reentrant circuits in the epicardial border zone of subacute canine infarcts," Circ. Res. 63, 182-206 (1988).

${ }^{141}$ I. Aranson, H. Levine, and L. Tsimring, "Controlling spatiotemporal chaos," Phys. Rev. Lett. 72, 2561-2564 (1994).

${ }^{142}$ S. M. Zoldi and H. S. Greenside, "Karhunen-Loève decomposition of extensive chaos,"' Phys. Rev. Lett. 78, 1687-1690 (1997).

${ }^{143} \mathrm{~T}$. Akiyama, "Intracellular recording of in situ ventricular cells during ventricular fibrillation," Am. J. Physiol. 240, H465-H471 (1981).

${ }^{144}$ J. F. Swartz, J. L. Jones, and R. D. Fletcher, "Characterization of ventricular fibrillation based on monophasic action potential morphology in the human heart," Circulation 87, 1907-1914 (1993).

${ }^{145}$ See AIP Document No. E-PAPS: E-CHAOE-8-025801 for original color movies. E-PAPS document files may be retrieved free of charge from our FTP server (http://www.aip.org/epaps/epaps.html) or from ftp.aip.org in the directory/epaps/. For further information, e-mail: paps@aip.org or fax: 516-576-2223. 